\title{
Das Wetter zu Ende des 18. Jahrhunderts
}

"Climatic changes are much discussed these days. That ist a sharp contrast to the situation forty or fifty years ago, when climatology was widely regarded as the dullest branch of meteorology - just the book keeping operation - and it was generally assumed that for all practical purposes climate could be treated as constant" (LAMB, 1982).

\section{Einleitung}

Klimageschichtliche Betrachtungen begannen in dem Zeitpunkt, als man meteorologische Beobachtungen einzelner Orte zu einem Gesamtbild vereinen wollte (BRANDES, 1820). Anstrengungen der «Societas Meteorologica Palatina», der "Société Nationale de Médecine» und vieler Einzelpersönlichkeiten vereinten eine Grundidee der Klimatologie: Man hoffte, durch räumliche Betrachtung verschiedener meteorologischer Meßwerte Einblick in die Ursachen von Witterungsveränderungen zu gewinnen.

In unserem Jahrhundert wurden punktuelle Neu- und Wiederbearbeitungen einzelner Säkularreihen und längerer Klimaaufzeichnungen aufgegriffen, jedoch wurde die Erstellung übersichtlicher Resultate erschwert durch das Fehlen wichtiger Informationen (Stations- und Instrumentengeschichte). Man konnte nur mit Mühe einen einheitlichen Vergleichsstand erreichen. So beschränkte man sich vorerst auf die Bearbeitung von Temperatur- und vereinzelten Luftdruckreihen (CLAYTON, 1927 und 1934; BIDER, SCHÜEPP, RUDLOFF, 1959 und 1961; BEHRENS, 1965; LAMB, 1966; LAUSCHER, 1981 etc.). Noch heute zeugen neueste Publikationen von den Unsicherheiten einer Bearbeitung solcher Klimareihen (ATTMANNSPACHER, 1981; KEIL, 1983).

In jüngster Zeit ist man zu räumlicher Betrachtungsweise übergegangen. Dies wurde möglich durch die nun vorhandene größere Stationsdichte und das wachsende Literaturangebot zum Thema Klimaänderung (LAMB, 1977 und 1982; PFISTER, 1984; ÖSCHGER, MESSERLI, SVILAR et al., 1980).

PFISTER (1984) gelang es, mit den sogenannten «Proxidaten» weit auf der Zeitachse zurückgreifende Klimainformation ohne direkte Messungen zu beschreiben. Seine Untersuchungen erlauben einen Rückschluß auf Sommertemperaturen oder Niederschlagsverhältnisse. Sie sind stark von den verwendeten Eichperioden abhängig.

Es erscheint wichtig, die instrumentellen Messungen möglichst weit auf der Zeitachse rückwärts auszuwerten. In dieser Arbeit bildet das Jahr 1781 den Startpunkt. Damals wurde durch die Initiative von Johann Jakob HEMMER unter KURFÜRST KARL THEODOR VON DER PFALZ ein einheitliches Meßnetz von maximal
38Stationen geschaffen. Das Netz bestand wohl nur bis 1792, dann wurden diese internationalen Bestrebungen durch die Französische Revolution beendet (ATTMANNSPACHER, 1981).

Eine Übersicht über das schweizerische Material findet sich im Anhang zu den Annalen der Schweizerischen Meteorologischen Anstalt (ANNALEN, 1957). Seither ist die Auswertung von Klimareihen aus Schaffhausen (1794-1845) und Zürich (1767-1802) dazugekommen (GISLER, 1983). Sie ermöglichte u.a. eine Kontrolle der Bearbeitung der Basler Reihe (BIDER, SCHÜEPP, RUDLOFF, 1959).

$\mathrm{Da}$ in den vergangenen Jahren durch PFISTER (1984) eine eingehende Sammlung und Verarbeitung aller bekannten, in den Archiven vorhandenen Daten erfolgte, dürfte das schweizerische Material zum größten Teil ausgeschöpft sein. Wir müssen versuchen, überregional mit Hilfe der ab 1781 möglichen Konstruktion mitteleuropäischer Wetterkarten Einblick in den Mechanismus der Wetterabläufe in der vorindustriellen Zeit während der «Kleinen Eiszeit» zu gewinnen.

\subsection{Wichtige Parameter}

Drei wichtige meteorologische Parameter bestimmen die Klimaverhältnisse in den letzten zwei bis drei Jahrhunderten, zu denen wir Meßresultate besitzen: Temperatur, Niederschlag und - als Grundlage zur Erforschung von Klimaschwankungen - der Luftdruck.

Wir beschäftigen uns im folgenden mit zwei grundlegenden Wetterelementen, welche bereits in früheren Jahrhunderten, seit der Entwicklung der Messinstrumente anfangs des 17. Jahrhunderts, registriert werden konnten: Lufttemperatur und Luftdruck.

Bis zum Jahr 1780 handelte es sich um vereinzelte Meß-Serien, welche selbst für die betreffenden Orte

Othmar Gisler, Dr. phil. II, auf Weinbergli 8, 6005 Luzern 
aus Mangel an Vergleichsstationen nur schwer zu einer homogenen Serie zusammengefügt werden können. RUDLOFF (1967) hat eine Übersicht über das vorhandene Material gegeben.

Für die Temperatur finden wir bei LAUSCHER (1981) in neuerer Zeit ebenfalls eine umfassende Zusammenstellung. Eine wesentliche Hilfe bilden die "Proxidaten", welche für die Schweiz für den Zeitraum von 1525-1963 von PFISTER (1984) zusammengestellt wurden. Noch weiter zurück reichen dendrochronologische, pollenanalytische und gletschergeschichtliche Daten, die jedoch mit Hilfe der modernen Messungen geeicht werden müssen (SCHWEINGRUBER, 1983; HOLZHAUSER, 1984).

Für die Niederschläge hat GISLER (1983) die Basler Reihe in bezug auf die Zahl der Tage mit Niederschlag näher untersucht. Auch die andern vorhandenen Reihen, insbesondere diejenigen von Genf (17681900), sind dort zusammengestellt. Es ergibt sich im Vergleich zwischen Schaffhausen und Genf ein bedeutender Unterschied, besonders von 1821-1830, wo Genf ein Minimum aufweist, während in Schaffhausen gleichzeitig das höchste Dezennienmittel der Reihe (1794-1845) auftritt. Ob hier eine Inhomogenität vorliegt oder beträchtlich große regionale Unterschiede auftraten, muß weiter abgeklärt werden. Beim Niederschlag sind solche Unterschiede im Gegensatz zu Temperatur und Luftdruck möglich.

\subsection{Meßnetz und Datenmaterial}

LAMB (1966) hat in den 1960er Jahren begonnen, globale Luftdruckverteilungen zu untersuchen. Unsere Arbeit beschränkt sich auf den Kontinent Europa und einen zeitlichen Ausschnitt aus der «Kleinen Eiszeib» von 1781-1792. Dieser Zeitabschnitt fällt mit der Aufbauphase von internationalen meteorologischen Meßnetzen im heutigen Sinne zusammen. In jenen Jahren wurde von Mannheim aus ein europäisches Meßnetz organisiert, betrieben und unterhalten (Societas Meteorologica Palatina, kurz: SMP).

1. Alle Beobachtungsinstrumente wurden von demselben Hersteller gefertigt und durch die Initianten an alle beteiligten Stationen gesandt.

2. Die Messungen wurden zu vorher genau festgelegten Zeitpunkten und nach einheitlichem Code abgefasst.

3. Jährlich wurden alle Meßergebnisse in ausführlicher Form publiziert (EPHEMERIDEN), 1781-1792.

Im mitteleuropäischen Raum finden wir - neben den zahlreichen Reihen in den Städten - eine seit 1781 (mit Ausnahme der Jahre 1811 und 1812) durchgehend vorhandene freigelegene Voralpenstation in ca. 1000 Metern Höhe auf dem Hohenpeissenberg in Bayern. Sie wurde durch GREBE (1957) eingehend bearbeitet. Anlässlich des 200-Jahr-Jubiläums 1981 wurden die Beobachtungen bis 1980 vervollständigt wiedergegeben, wobei auf eine Homogenisierung verzichtet wurde (ATTMANNSPACHER, 1981). Auf den Unterschied beider Bearbeitungen hat KEIL 1983 hingewiesen.

$\mathrm{Zu}$ Beginn der Messreihen hatte man mit großen instrumentellen Schwierigkeiten zu kämpfen (z. B. bei den Thermometern mit der Alterung des Glases mit entsprechender Nullpunktsverschiebung. Vgl. GISLER, 1983, S. 15/16, 52). Nach Einführung der Jenaer Gläser blieben noch Unterschiede in der Aufstellung (Hausaufstellung, Wildsche Hütte, Stevenson Screen). Für eine Verarbeitung der Luftdruck- und Temperaturwerte zu monatlichen Mittelkarten mussten die Stationshöhen (Barometerhöhen) und Koordinaten der Einzelstationen aus verschiedenen Quellen beschafft werden. Die EPHEMERIDEN enthielten größtenteils kurze Stationsbeschreibungen inklusive Höhenangaben und Koordinaten. Waren sie unvollständig oder fehlten sie, mußte auf Atlanten und weitere Handbücher zurückgegriffen werden. Für die Lage der Station spielte es keine Rolle, die Koordinaten in Graden östlich des Monte Teide (Teneriffa), östlich von Paris

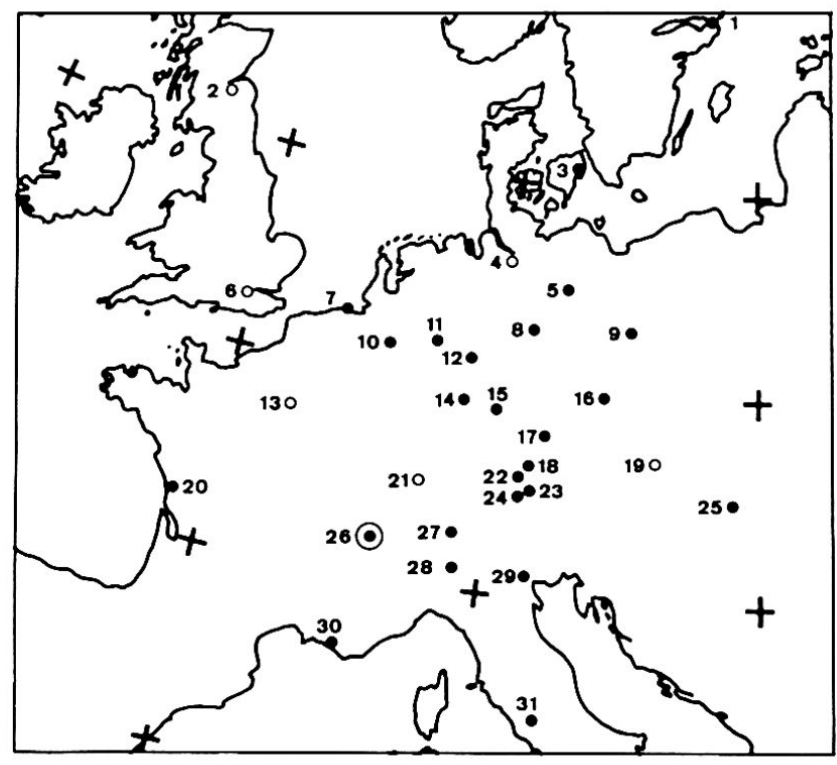

Figur 1 Für diese Untersuchung benutzte Stationen aus dem Meßnetz der Societas Meteorologica Palatina und aus neueren Publikationen

\begin{tabular}{|c|c|c|}
\hline 1 Stockholm & 11 Bonn & 21 Basel \\
\hline 2 Edinbourgh & 12 Göttingen & 22 Hohenpeißenberg \\
\hline 3 Kopenhagen & 13 Paris & 23 Tegernsee \\
\hline 4 Hamburg & 14 Mannheim & 24 Andechs \\
\hline 5 Berlin & 15 Würzburg & 25 Budapest \\
\hline 6 London & 16 Prag & 26 Genf \\
\hline 7 Middlebourg & 17 Regensburg & 27 St. Gotthard \\
\hline 8 Erfurt & 18 München & 28 Mailand \\
\hline 9 Sagan & 19 Wien & 29 Padua \\
\hline 10 Brüssel & 20 La Rochelle & $\begin{array}{l}30 \text { Marseilles } \\
31 \text { Rom }\end{array}$ \\
\hline
\end{tabular}

- Daten der Societas Meteorologica Palatina O Neuere publizierte Daten 
oder weiteren Nullmeridianen vorzufinden (ab insula ferro computato beispielsweise). Heutige Kartenwerke lassen diese Örtlichkeiten schnell bestimmen.

Eher Kopfzerbrechen bereitet jeweils die Höhenbestimmung für die damalige Stationslage. Zusammen mit folgenden Punkten bilden sie die Hauptprobleme einer Berechnung von Luftdruckkarten:

1. Die Luftdruckeinheiten waren nicht mit heutigen identisch. Es können umrechnungsbedingte Lükken entstehen (Ablesegenauigkeit 0,3 bis $0,4 \mathrm{hPa}$, vgl. GISLER 1983, Seite 59 ff.).

2. Die historischen Höhenangaben sind teils unsicher.

3. Die für die Reduktion auf $0^{\circ} \mathrm{C}$ nötige Angabe der Quecksilber- oder Raumtemperatur war nicht immer vorhanden.

Bei der Arbeit mit den Quecksilbertemperaturen der SMP-Stationen schälten sich drei Gruppen heraus:

1. Stationen, auf denen das Barometer in Räumen untergebracht war, welche bei Aussentemperaturen tiefer als ca. $10-12^{\circ} \mathrm{C}$ durch Heizen eine Raumtemperatur von $12-15^{\circ} \mathrm{C}$ aufrecht hielten.

2. Stationen mit schwach beheizten $\left(\leqq 12^{\circ} \mathrm{C}\right)$ Räumen für das Barometer.

3. Stationen mit ungeheizten Räumen für das Barometer (Figur 2).

\section{Zimmer-}
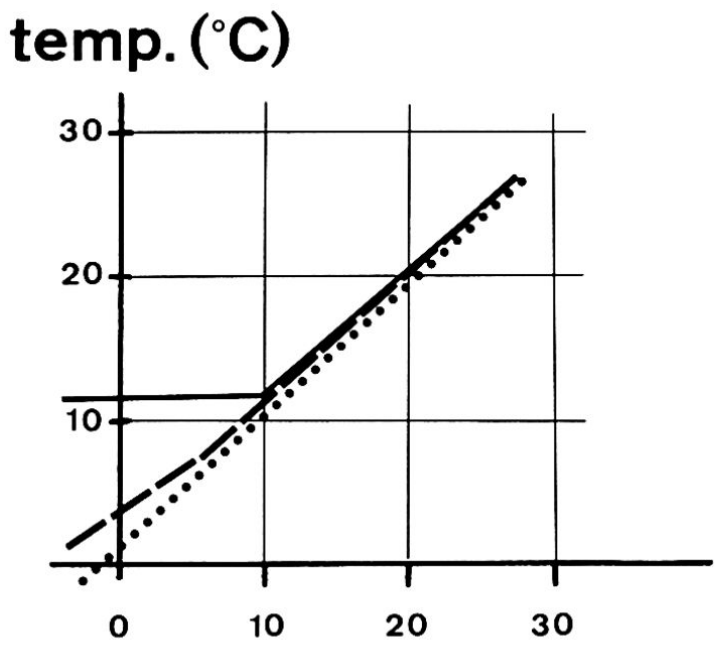

Aussentemperatur $\left({ }^{\circ} \mathrm{C}\right)$

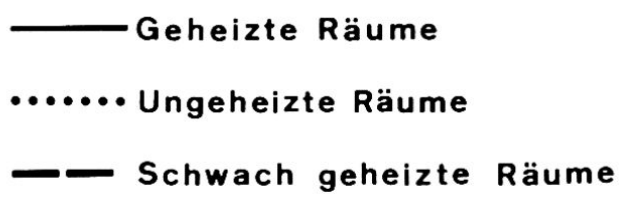

Figur 2 Beziehung zwischen Zimmer- und Außentemperatur in verschieden geheizten Räumen auf Stationen der Societas Meteorologica Palatina

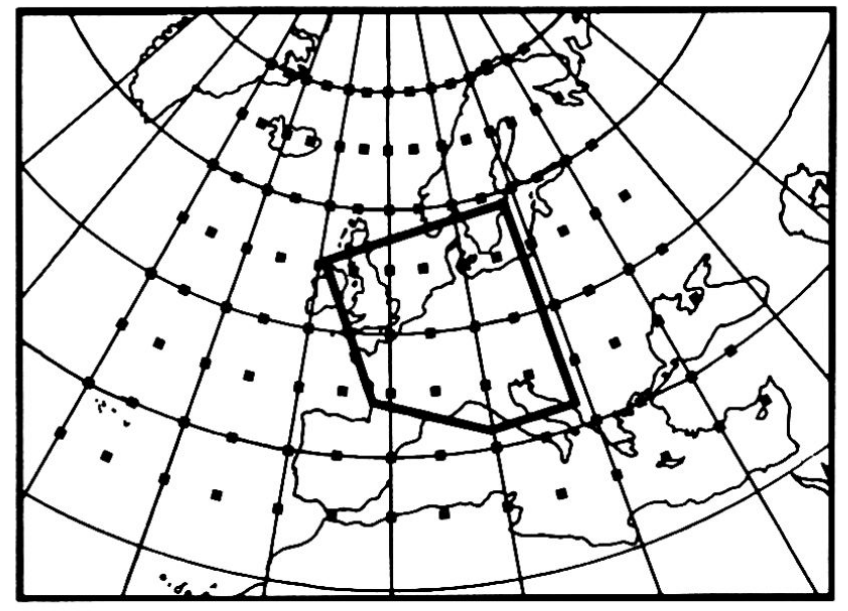

Figur 3 Gitterpunktnetz der Luftdruckwerte nach KINGTON und für diese Bearbeitung ausgeschiedene Kernregion

Nach verdankenswerter Vermittlung durch Prof. H.H. LAMB erhielten wir wertvolle Unterstützung in Form von Luftdruckwerten an regelmäßig über Europa verteilten Gitterpunkten (Figur 3).

Diese Daten wurden durch J.A. KINGTON 1978 in Bearbeitung vorgestellt. Er zeichnete für die Jahre 1781-1785 tägliche synoptische Wetterkarten. Die dazugehörigen Stationsgeschichten und weitere wertvolle Informationen konnten anlässlich eines persönlichen Aufenthaltes bei H.H. LAMB für diese Arbeiten benutzt werden. Für die täglichen Wetterkarten benutzte KINGTON Daten von über 50 Stationen einschließlich jener der SMP. Daraus wurden Luftdruckwerte an einzelnen Gitterpunkten berechnet. Für ein Ausarbeiten von monatlichen Luftdruckkarten standen uns pro Gitterpunkt nicht immer gleich viele Werte zur Verfügung. Mit der in Figur 4 gezeigten Belegungsdichte eines einzelnen Gitterpunktes ist die Aussagekraft darauf basierender Karten direkt korreliert.

In unserer Bearbeitung verwendeten wir diese Daten nebst jenen der SMP-Stationen aus den EPHEMERIDEN und anderweitig schon bearbeiteter und publizierter Reihen (CLAYTON, 1927, 1934; KREIL, 1854; RENOU, 1881; BEHRENS, 1965; BIDER, SCHÜEPP, 1959 und 1961). Der Datensatz von KINGTON hatte den Vorteil, mit heutigen Luftdruckwerten von 1945-1980 an denselben Gitterpunkten verglichen werden zu können (Druckdaten aus der Alpenwetterstatistik der Schweiz. Meteorologischen Anstalt). Ein wesentlicher Nachteil wurde mit der grafischen Darstellung der Druckkarten mittels Computer erkannt. Im Alpenbereich zeigte sich die zu große Maschenweite des Gitternetzes. Besonders Nordsüdgradienten wurden verfälscht wiedergegeben als Folge einer linearen Interpolation der Druckwerte zwischen den Gitterpunkten (Figur 5). Weiter waren die Karten in den Randgebieten bei stärker auftretenden Gradienten weniger wirklichkeitsnah. 
Solchen Effekten mußte mit genauerem Studium der Druckverhältnisse anhand der Alpenrandstationen Basel, Genf und Mailand begegnet werden. Für unsere Karten wurde eine kleinere, besser abgesicherte Kernregion ausgeschieden (Figur 3).

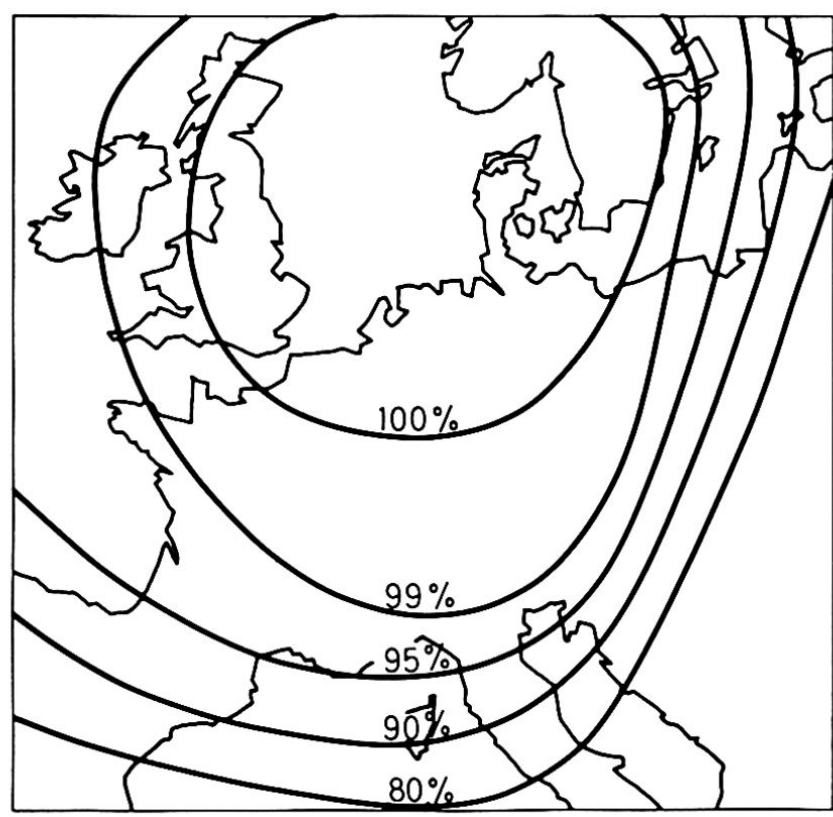

Figur 4 Prozentuelles Auftreten von Luftdruckwerten pro Gitterpunkt

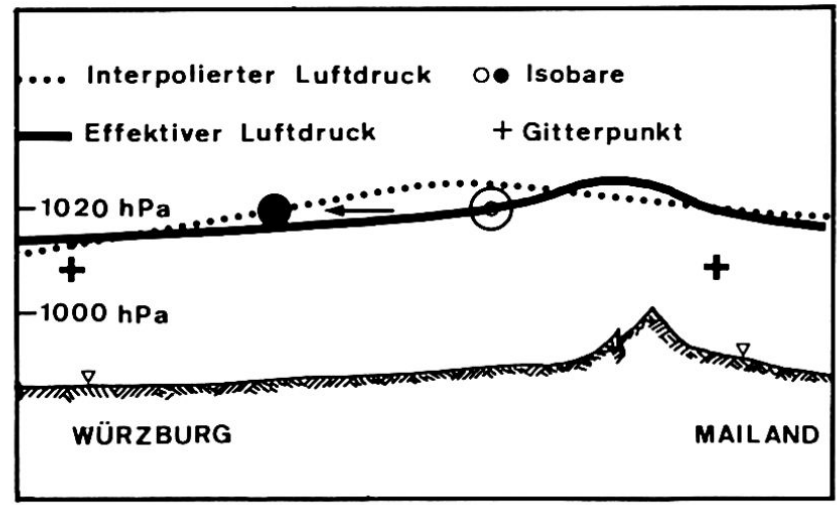

Figur 5 Verschiebung von interpolierten Isobaren über den Alpen aufgrund eines zu groben Gitternetzes der Luftdruckwerte

\section{Das Wetter Ende 18. Jahrhundert}

\subsection{Luftdruck- und Temperaturkarten}

Für die hier wiedergegebenen Temperatur- und Luftdruckkarten dienten die Meßwerte an rund 20 Stationen des Pfälzischen Netzes (SMP-Werte), die durch KINGTON berechneten Gitterpunktwerte des Luftdruckes (KINGTON-Werte), und die Luftdruck- und/oder Temperaturreihen von Edinburgh, Paris, Basel, Genf, Mailand, Wien, Hamburg und Berlin (publizierte Werte).

Aus den SMP-Werten für Luftdruck und Temperatur allein ließen sich kaum vernünftige Bodenluftdruckkarten herstellen. Dagegen konnten Temperaturübersichten und Karten der thermischen und barometrischen Unruhe ohne große Hindernisse rasch gezeichnet werden. Als thermische oder barometrische Unruhe bezeichnen wir Analysen der Änderung des entsprechenden Elementes von Monat zu Monat (z. B. Februarluftdruck - Januarluftdruck $=\Delta$ P Februar).

Da uns über die SMP-Daten weitgehend alle nötigen Informationen zur Reduktion des Luftdruckes auf 0 Meter Höhe und $0^{\circ} \mathrm{C}$ zur Verfügung standen, konnten die KINGTON-Werte ebenfalls einer solchen Reduktion unterzogen werden. Die ursprünglichen KINGTONWerte boten in zweierlei Hinsicht Probleme:

\section{Kartenrandproblem:}

Ungeachtet der Belegungsdichte wurden durch den Computer Karten gezeichnet; dies verlangte ein zweites, sorgfältiges Nacharbeiten von Hand in einer kleineren Kernregion (vgl. LAMB, 1966; JONES, WIGLEY, BRIFFA, 1983 und Figur 3).

\section{Reduktionsproblem und Gradientbereinigung:}

Durch die recht genau durchführbare Reduktion der SMP-Werte wurde es möglich, die mittels einer Konstanten auf $0^{\circ} \mathrm{C}$ und Null Meter Höhe reduzierten KINGTON-Werte genauer von ihrer temperaturbedingten Abweichung zu Vergleichswerten zu befreien.

Für tägliche synoptische Karten reicht eine Reduktion des Luftdruckes mittels einer Konstanten aus. Die hier gezeigten monatlichen Übersichten wurden hingegen genauer reduziert.

Mit Tabelle 1 möchten wir vorab einer Besprechung der Temperatur- und Luftdruckkarten anfügen, daß zwischen den Mittelwerten der beiden Elemente für die Jahre 1781-1788 und dem vollständigen Dezennium kein wesentlicher Unterschied besteht.

$\mathrm{Da}$ die Temperaturkarten ausschließlich aus den Monatszusammenstellungen des SMP-Netzes gewonnen wurden, fehlten die Angaben für das Jahr 1782. Mit Hilfe der publizierten Werte kann jedoch gezeigt werden, $\mathrm{da} ß$ das Temperaturmittel durch Hinzufügen dieses Jahres höchstens noch mehr erniedrigt würde. Die Luftdruckkarten decken alle Jahre vollständig ab.

\subsection{Vergleiche mit heute}

In Mittel- und Nordeuropa war es $1781-1788$ bis $2{ }^{\circ} \mathrm{C}$ kälter als heute (1931-1960) bei allgemein gleichem bis leicht höherem Luftdruck. Die saisonalen Temperaturkarten zeigen vor allem kältere Winter in Nordwest- bis Nordeuropa. Die Sommer waren gleich warm oder nur leicht wärmer (Stadteinfluß, keine Freilandstationen). Der Luftdruck war im Winter über 
Tabelle 1 Vergleich der Mittelwerte der Lufttemperatur und des Luftdruckes einiger europäischer Orte in zwei Zeitabschnitten

\begin{tabular}{|c|c|c|c|c|c|c|}
\hline \multicolumn{7}{|c|}{ Temperatur (Grad Celsius) } \\
\hline & Edinburg & Centr.-England & Paris & Mailand & Wien & Berlin \\
\hline $\begin{array}{l}\text { A: } 1781-88 \\
\text { B: } 1781-90\end{array}$ & $\begin{array}{l}8,13 \\
8,27\end{array}$ & $\begin{array}{l}8,83 \\
8,89\end{array}$ & $\begin{array}{l}10,18 \\
10,24\end{array}$ & $\begin{array}{l}12,79 \\
12,76\end{array}$ & $\begin{array}{l}8,97 \\
9,12\end{array}$ & $\begin{array}{l}8,43 \\
8,59\end{array}$ \\
\hline$A-B$ & $-0,14$ & $-0,06$ & $-0,06$ & 0,03 & $-0,15$ & $-0,16$ \\
\hline \multicolumn{7}{|c|}{ Luftdruck $(1000+\ldots \mathrm{hPa})$} \\
\hline & Edinburg & Centr.-England & Paris & Mailand & Wien & Berlin \\
\hline $\begin{array}{l}\text { A: } 1781-88 \\
\text { B: } 1781-90\end{array}$ & $\begin{array}{l}13,09 \\
12,41\end{array}$ & $\begin{array}{l}- \\
-\end{array}$ & $\begin{array}{l}18,16 \\
18,56\end{array}$ & $\begin{array}{l}14,50 \\
14,53\end{array}$ & $\begin{array}{l}19,00 \\
19,02\end{array}$ & $\begin{array}{l}- \\
-\end{array}$ \\
\hline$A-B$ & 0,68 & & 0,04 & $-0,03$ & $-0,02$ & \\
\hline
\end{tabular}

der Nordsee, im Frühling über England und Frankreich, im Herbst über Westeuropa höher als heute.

Besonders die Temperaturabweichungen nach Figur 6 lassen eine Verschiebung der historischen Jahreszeiten vermuten. Durch eine Vorverlegung von Sommer und Herbst und eine Verlängerung des Winters blieb wenig Raum für eine frühlinghafte Erwärmung oder luftdruckmäßige Vorbereitung auf die Sommerzirkulation.

Eine stetige Luftdruckabnahme im Bereich der Nordsee und Südnorwegens/-schwedens in den Monaten Januar bis März macht deutlich, daß damals aus dem Sektor NW kalte Polarluft kräftig auf den Kontinent fließen konnte. Dies ergab über der Nordsee mittlere Lufttemperaturen von $0^{\circ} \mathrm{C}$; heute liegt diese Temperaturlinie zur gleichen Jahreszeit nördlich von Island. Die anschließende Druckerhöhung von April bis in den August ist Ausdruck eines kräftiger nach Europa reichenden Azorenhocheinflusses bei gleichzeitig sich gegen den Herbst hin zuspitzenden Gradienten zwischen Mittel- und Nordeuropa (Stürme, vgl. LAMB, 1984). Es handelte sich um eine Verstärkung der Westwindzirkulation, nicht unbedingt um eine Nordsüdverschiebung der Drucksysteme, wie sie FAY 1958 angenommen hat.

Eine verstärkt angenommene Strömung aus SW bis NW brachte für Norddeutschland im September im Mittel wärmere Luft auf den Kontinent als heute. Der herbstliche Hochdruckrücken war über den Alpen etwas verstärkt. Schon im Oktober schalteten die Verhältnisse wieder auf Winter um. Von Frankreich bis zur Ostsee war es im Schnitt $1^{\circ} \mathrm{C}$ kälter als heute. Dieser Trend setzte sich bis in den Dezember verstärkt fort, so daß in Zentralengland Temperaturen herrschten wie heute zwischen Island und Norwegen. In Dänemark sanken sie unter den Gefrierpunkt, während die Temperaturen heute dort im Dezember bei $3^{\circ} \mathrm{C}$ liegen.

Der Luftdruck zeigte ab Oktober ein sich verstärkendes winterliches Hoch über den Alpen und Westeuropa. Heute bildet sich über der Alpenregion lediglich ein Ausläufer des russischen Hochs. Die antizyklonale Ausprägung des historischen Druckfeldes ist offensichtlich. Verstärkt erscheint auch das herbstliche Hoch über den Alpen und vor allem die mittlere Drucksituation im Frühling. Sie ist als Ausdruck des verlängerten Winters anzusehen. Die Sommerdruckverhältnisse entsprechen sich in ihrer Ausprägung, nicht aber in ihrer absoluten Höhe.

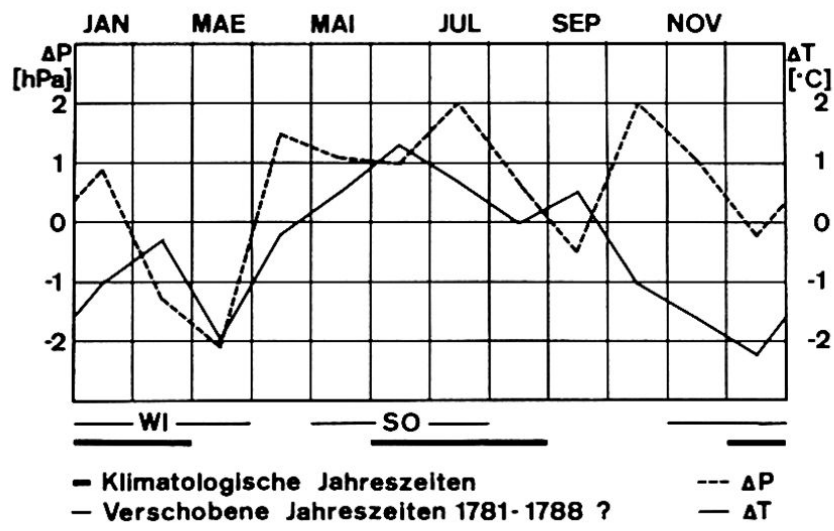

Figur 6 Abweichungen der mittleren Monatsmittel des Luftdruckes und der Lufttemperatur der Jahre 1781-1788 von jenen der Periode 1931-1960 (Mittel 1781-88 minus Mittel 1931-60)

Tabelle 2 Abweichungen der mittleren Monatsmittel des Luftdruckes und der Lufttemperatur der Jahre 1781-1788 von jenen der Periode 1931-1960 (Mittel 1781-1788 minus Mittel 1931-1960, Mittelwerte aus den Luftdruckwerten der Kernregion)

\begin{tabular}{ccccccccccccc}
\hline Jan. & Feb. & März & April & Mai & Juni & Juli & Aug. & Sept. & Okt. & Nov. & Dez. \\
\hline 0,9 & $-1,3$ & $-2,1$ & 1,5 & 1,1 & 1,0 & 2,0 & 0,2 & $-0,5$ & 2,0 & 1,1 & $-0,2$ & $\mathrm{hPa}$ \\
$-1,0$ & $-0,3$ & $-1,9$ & $-0,2$ & 0,5 & 1,3 & 0,7 & 0,0 & 0,5 & $-1,0$ & $-1,6$ & $-2,2$ & $\mathrm{C}$ \\
\hline
\end{tabular}




\subsection{Winde auf dem Hohenpeißenberg und die Wärme- verhältnisse im Donauraum}

Aus den Karten wird eindeutig ersichtlich, wie die winterlichen Druckzustände sich gegenüber heute frühzeitig einstellten, aber auch später endeten. Als einschneidendste Veränderung kann der verlängerte Winter und das Fehlen eines eigentlichen Frühlings bezeichnet werden (Figur 6).

RUDLOFF beschrieb 1967 die Windrichtungen auf dem Hohenpeißenberg. Auffallend hohe Anteile an SSEWinden, aber sehr kleine Häufigkeiten an WSWRichtungen waren für die Zeit von 1781-1800 charakteristisch. Für die Zeit von 1781-1790 fand LAUSCHER 1980 und 1981, daß dieses Dezennium im Mittel von 21 europäischen Stationen um $0,22^{\circ} \mathrm{C}$ kälter war als die Mitteltemperatur von 1781-1979, der Donauraum jedoch um $0,4^{\circ} \mathrm{C}$ wärmer. Diese Temperaturanomalie sei in den Jahren 1791-1810 am größten gewesen. Diese sogenannte "Wärmeinsel im Donauraum» und die verstärkt auftretenden Winde auf dem HohenpeiBenberg könnten die Vermutung auftauchen lassen, im Donauraum hätte eine vermehrte Föhntendenz geherrscht.

Verschiedene Gründe sprechen dagegen. Einmal zeigen die saisonalen und monatlichen Luftdruckverteilungen für jenen Raum keine diesen Sektor bevorzugenden Lagen. Die in Tabelle 3 widergegebenen Windverhältnisse auf dem Gotthardpaß zeigen keine wesentlich andere Windverteilung für die historische Periode.

Eine Auszählung der Originalwindbeobachtungen auf dem Hohenpeißenberg für die Jahre 1783-1788 ergab eine größere Häufigkeit der Windrichtungen um SE verglichen mit heute. Dabei zeigte sich aber auch, daß es sich vorwiegend um Schwachwinde gehandelt hat (ca. 70\%, vgl. Figur 7). Die Windregistrierung kann durch kleine lokale Einflüsse stark veränderte Resultate zeigen. Eventuell sind sie von einer mechanisch bevorzugten Nullstellung der Windfahne beeinflußt. Auffallend ist jedoch die absolute Zunahme der
Windrichtungen aus den Sektoren Nord und Ost bei gleichzeitiger Erhöhung des prozentuellen Anteils der Starkwinde (Tabelle 3, Figur 7). Dies unterstreicht die größere Häufigkeit von stärkeren Nord- und Bisenlagen in der Palatina-Zeit.

Die Temperaturkarten vermitteln einen Eindruck, der zumindest für die Zeit von 1781-1790 kaum Platz frei

\section{Anteil Schwachwinde}

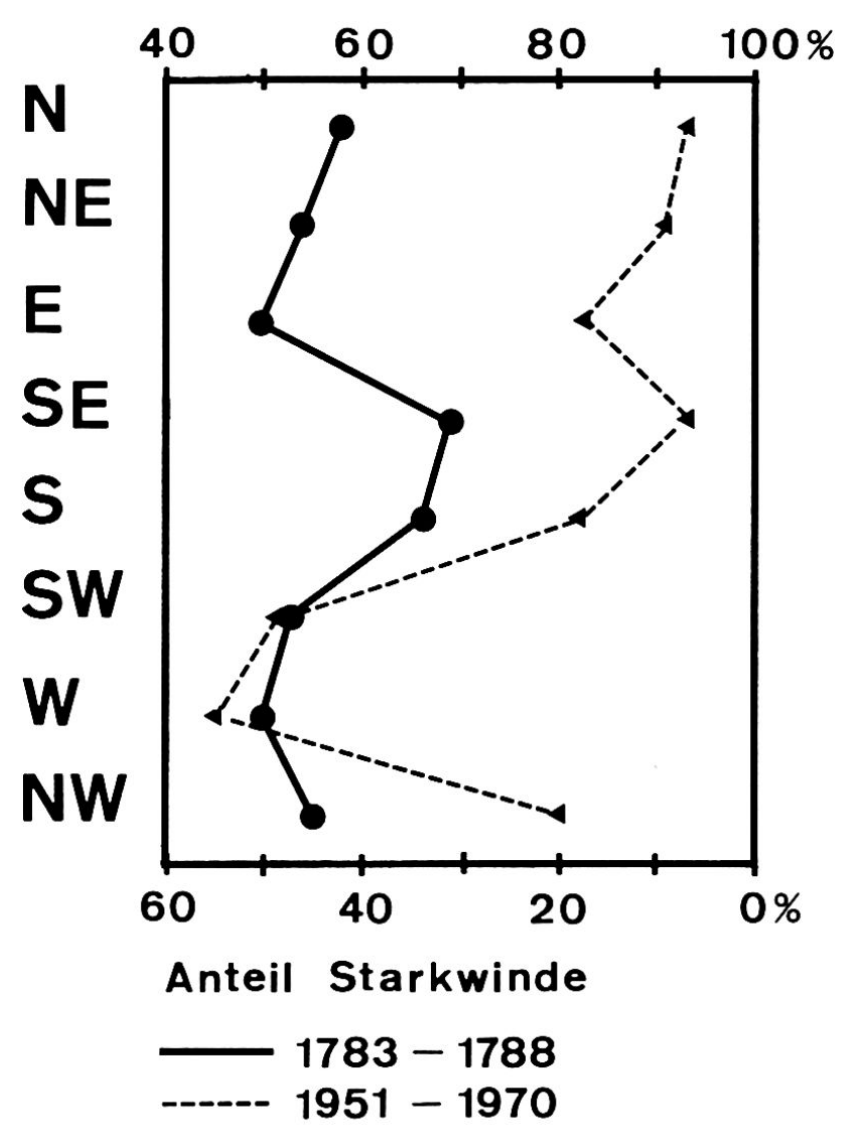

Figur 7 Prozentueller Anteil der Schwachwinde (0-3 Beaufort) an der Gesamthäufigkeit einzelner Windrichtungen auf dem Hohenpeißenberg von 1783-1788 und von 1951-1970

Tabelle 3 Prozentuelle Häufigkeiten der Windrichtungen auf dem Gotthard und dem Hohenpeißenberg

\begin{tabular}{|c|c|c|c|c|c|c|}
\hline \multirow[b]{2}{*}{ Richtung } & \multicolumn{2}{|l|}{ Gotthard } & \multicolumn{4}{|c|}{ Hohenpeißenberg } \\
\hline & $1903-1960$ & $1783-1788$ & $1903-1960$ & $1951-1970$ & 1783 & 788 \\
\hline NW & $15,8)$ & $55,2)$ & $6,7)$ & $6,9)$ & 8,0 & \\
\hline $\mathrm{N}$ & 38,7 & 0,3 & 4,9 & 6,8 & 3,2 & \\
\hline NE & 83,6 & 83,9 & 45,0 & 47,8 & 15,4 & 51,06 \\
\hline$E$ & 16,0 & 4,0 & 15,0 & 16,4 & 12,4 & \\
\hline SE & $22,2)$ & $24,4)$ & $4,0)$ & $5,4)$ & 12,0 & \\
\hline S & 15,8 & 14,1 & 3,4 & 5,5 & 10,2 & \\
\hline SW & 0,3 & 0,8 & 16,6 & 16,0 & 16,0 & \\
\hline w & 0,3 & 1,2 & 34,4 & 30,7 & 22,8 & \\
\hline
\end{tabular}




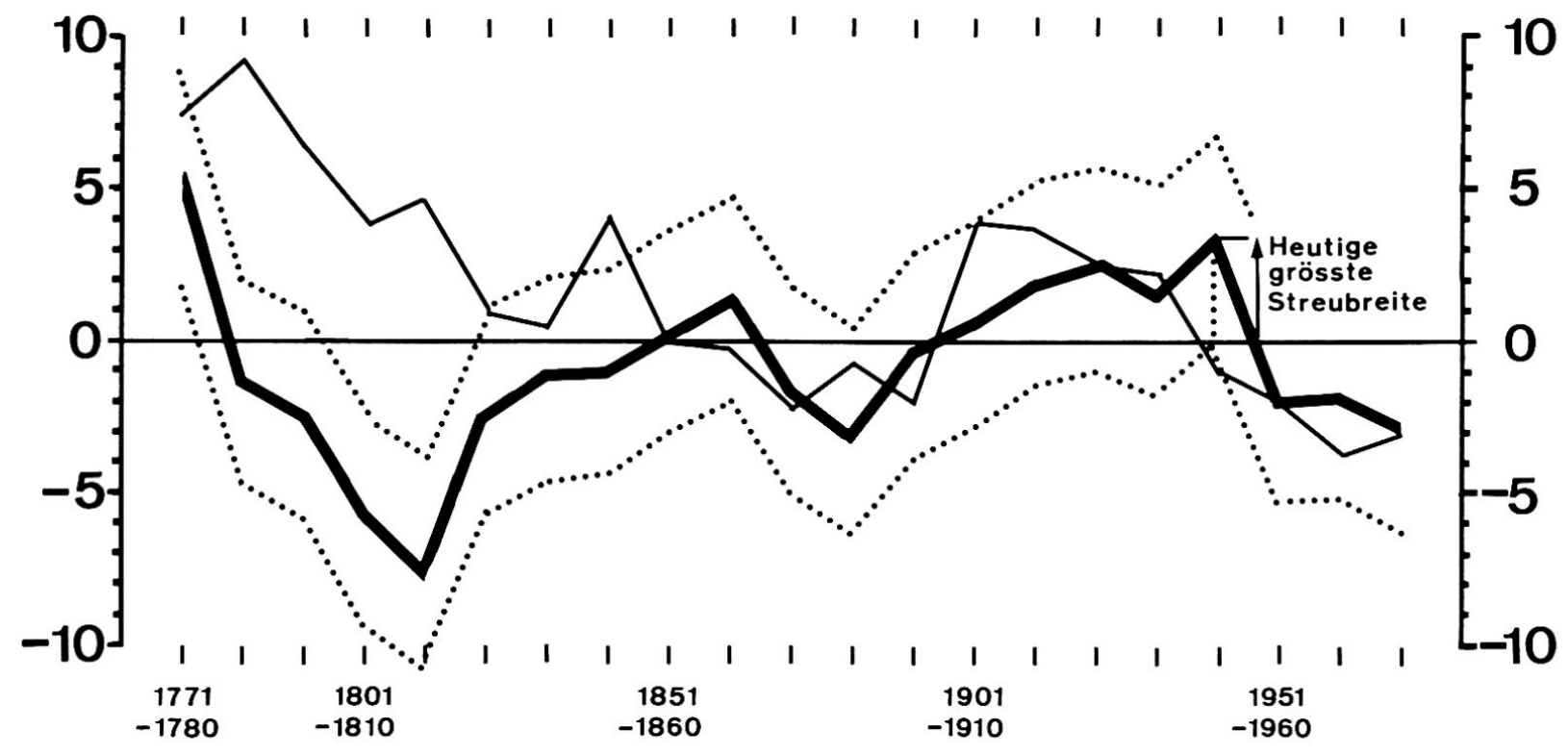

$\Delta T_{1}: T($ Kremsmünster $)-T(W i e n)$

$\Delta T_{3}$ : $T($ Kremsmünster $)-T(B a s e l)$

$\Delta T_{2}: T(B a s e l)-T($ Wien $)$

$\frac{\Delta T_{1}-\Delta T_{2}}{2}=$

Berechnete Differenz $\Delta T_{3}$

Figur 8 Verlauf der berechneten und gemessenen Temperaturdifferenz der Dezennienmittel: Kremsmünster-Basel

läßt für wesentlich vermehrte Erwärmung durch Föhn im Donauraum. Aus der Differenzbildung mittels Dezennienmittel der Temperatur umliegender Stationen kann vermutet werden, daß die Temperaturreihen von Wien und Kremsmünster einer weiteren Bearbeitung unterzogen werden sollten (Figur 8).

Die Vermutung, die Basler Temperaturen selber lägen zu tief, läßt sich anhand eines Vergleichs mit den Mittelwerten von 1951 bis 1980 entkräften (Tabelle 4). Mit einer solchen Annahme würden sich die Temperaturen aus Basel zu sehr dem heutigen Niveau nähern.

\section{Resultate und Ursachendiskussion}

Die Unterschiede der Luftdruckverhältnisse gegenüber heute zeigen sich eindrücklich bei der Darstellung der Veränderungen von Monat zu Monat. Die Häufigkeitsverteilungen der historischen und heutigen barometrischen Unruhen sind verschieden (vgl. Seite 5). So zeigt die heutige Verteilung ein ruhigeres Bild, d. h. man findet häufig Änderungen mit kleinen positiven oder negativen Beträgen. Die barometrische Unruhe der 1780 er Jahre ergab größere Wechsel, die monatlichen Druckänderungen waren im Mittel abrupter (Figur 9). Zwischen verlängerten Winterverhältnissen und etwa gleichlangen Sommern und Herb- sten geschah die Umstellung von einer Hauptsaison zur andern heftiger und in kürzerer Zeit.

Der früh einsetzende Aufbau des winterlichen Hochs über Ost- und Mitteleuropa schon im Oktober und die verlängerten Winterzustände bis in den März führten praktisch zu einem Ausfallen des Frühlings.

Temperaturmäßig wichen die Übergangsmonate am stärksten von der heutigen Situation ab. In einem Streifen von England nach der Ostsee fand die größte Abkühlung statt. Die Sommertemperaturen waren praktisch unverändert. Die meisten Stationen machten ihre Messungen innerhalb von Städten. Ein deshalb zu veranschlagender Stadteinfluß (mit einer Erwärmung von ca. $0,4^{\circ} \mathrm{C}$ ) und die damals noch üblichen Dreiermittelberechnungen (ca. $0,3^{\circ} \mathrm{C}$ zu hoch) mögen vor allem im Sommer dafür verantwortlich sein, daß die historischen Temperaturen als noch leicht zu hoch erscheinen mögen. Eindeutig durch mehrere Temperaturreihen belegbar ist der oben geschilderte verlängerte und "kernlose» Winter, wie er heute noch in extremer Ausbildung im Südpolargebiet zu finden ist (Figur 10).

Wie LAMB 1982 darlegt, bildeten die 20 Jahre von 1781 bis 1800 das Maximum der Küstenvereisung Islands (Figur 11). Mehr als die Hälfte des Jahres war die Ostküste Islands vereist. Durch die nach Süden vorgeschobenen Eismassen konnte Arktikluft öfter und intensiver auf den Kontinent gelangen (vgl. Luftdruckmittelwerte im Frühling und die damit verbundene Strömung). 

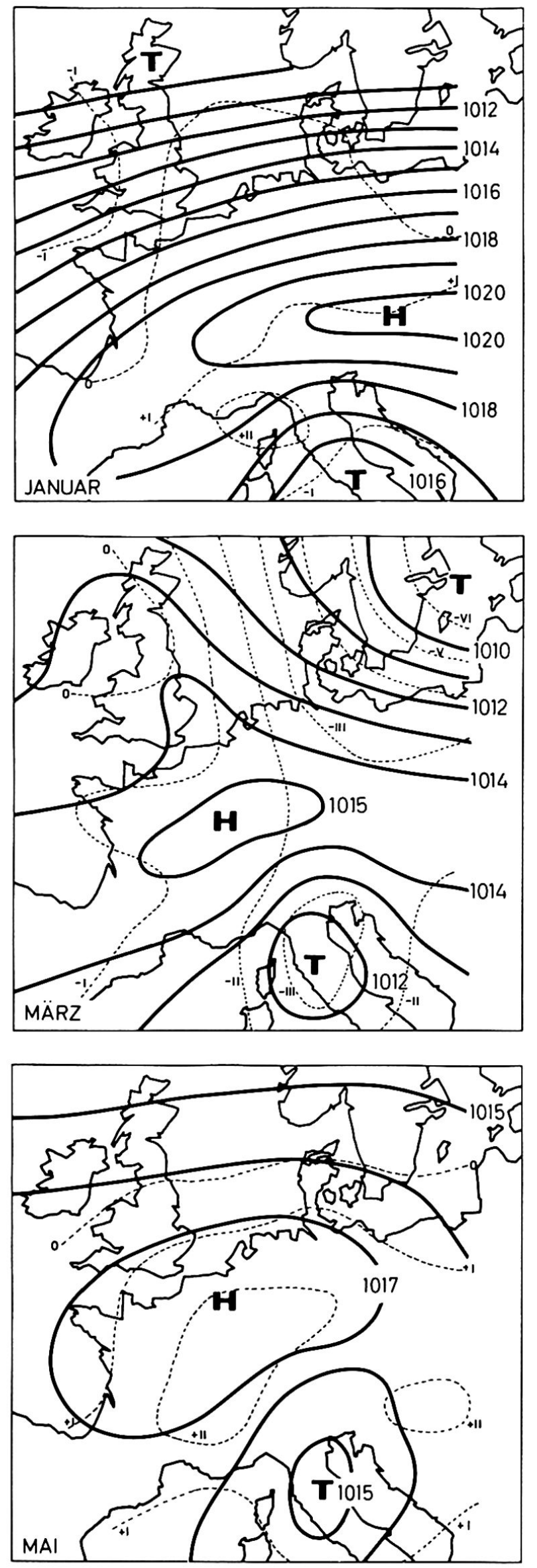
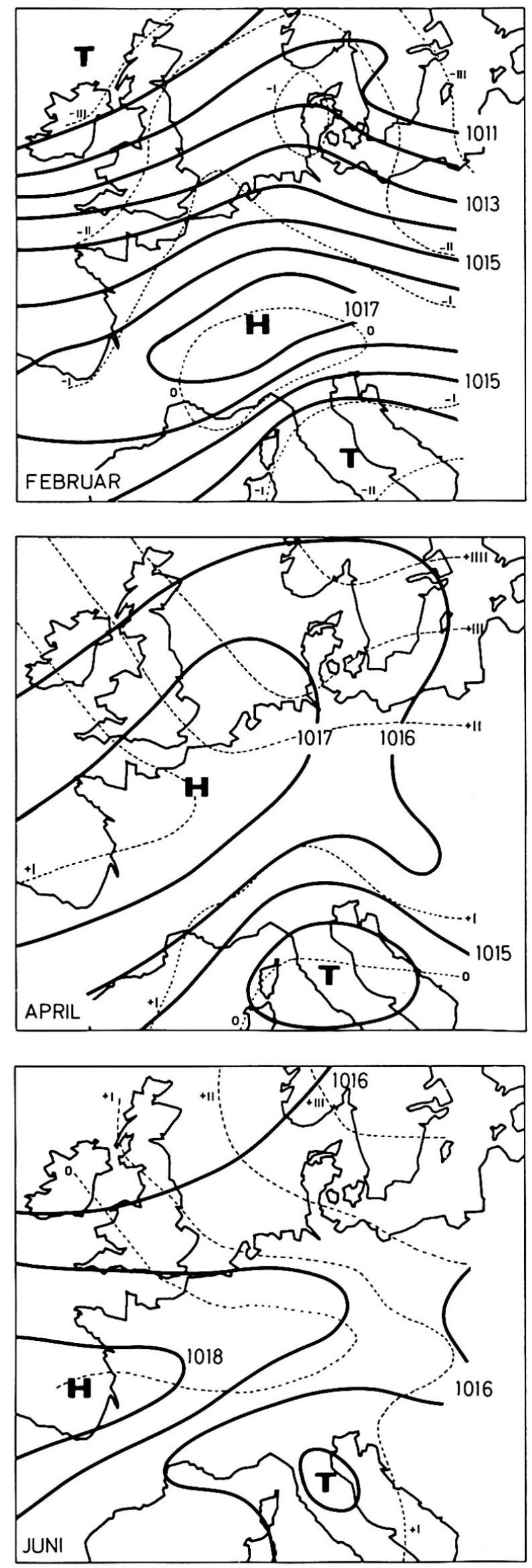

Monatliche Mittelkarten der Luftdruckverteilung über Mitteleuropa 1781-1788 mit den Abweichungen vom Mittel $1931-1960$ (hPa, auf Meereshöhe reduziert) 

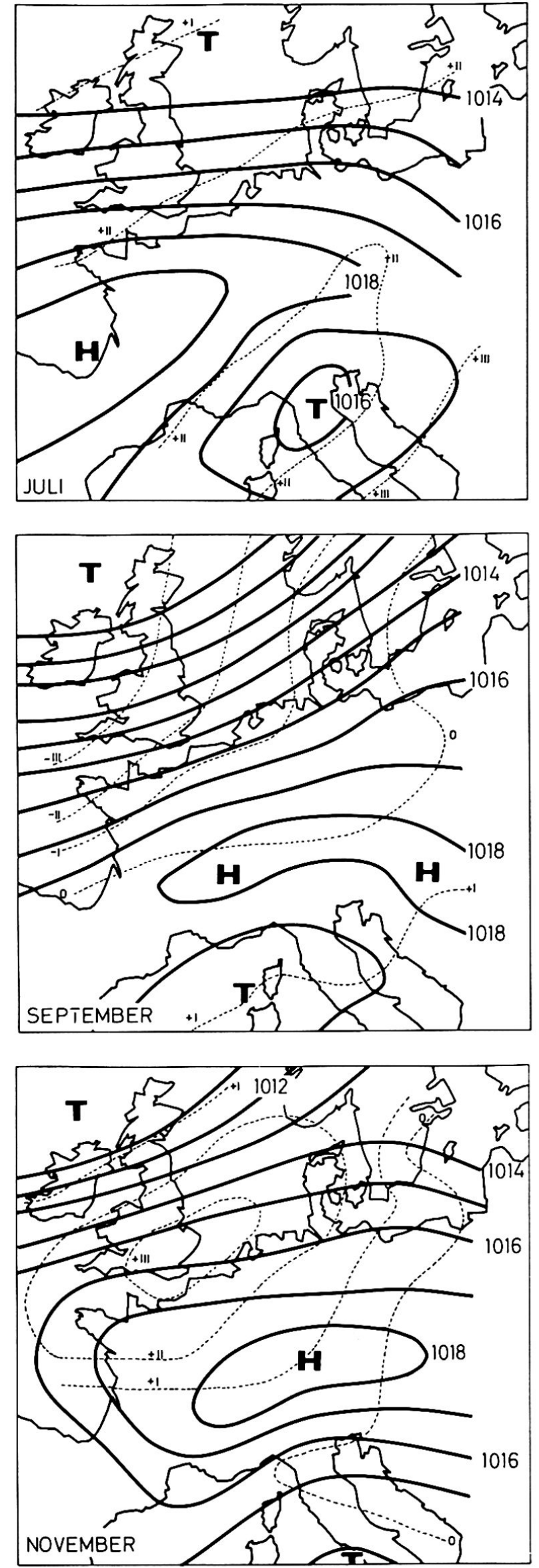
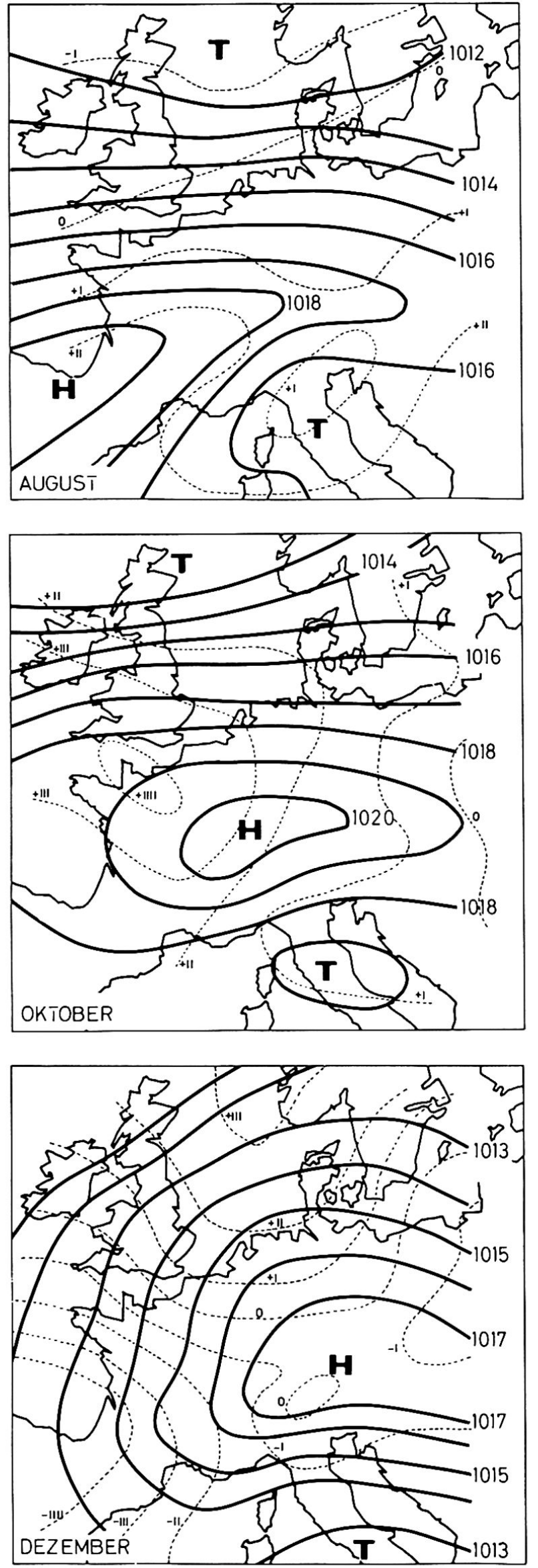

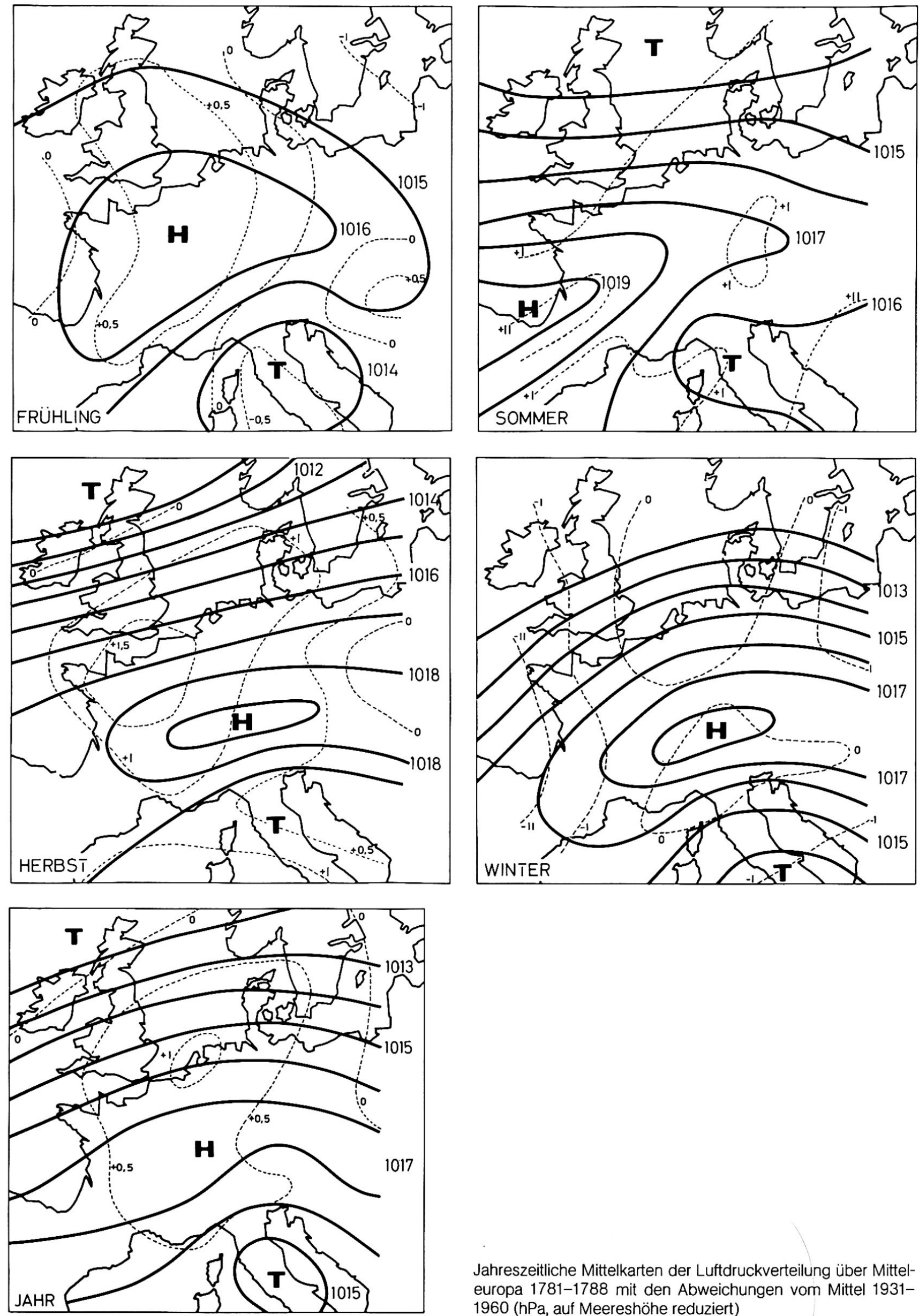

Jahreszeitliche Mittelkarten der Luftdruckverteilung über Mitteleuropa 1781-1788 mit den Abweichungen vom Mittel 19311960 ( $\mathrm{hPa}$, auf Meereshöhe reduziert) 

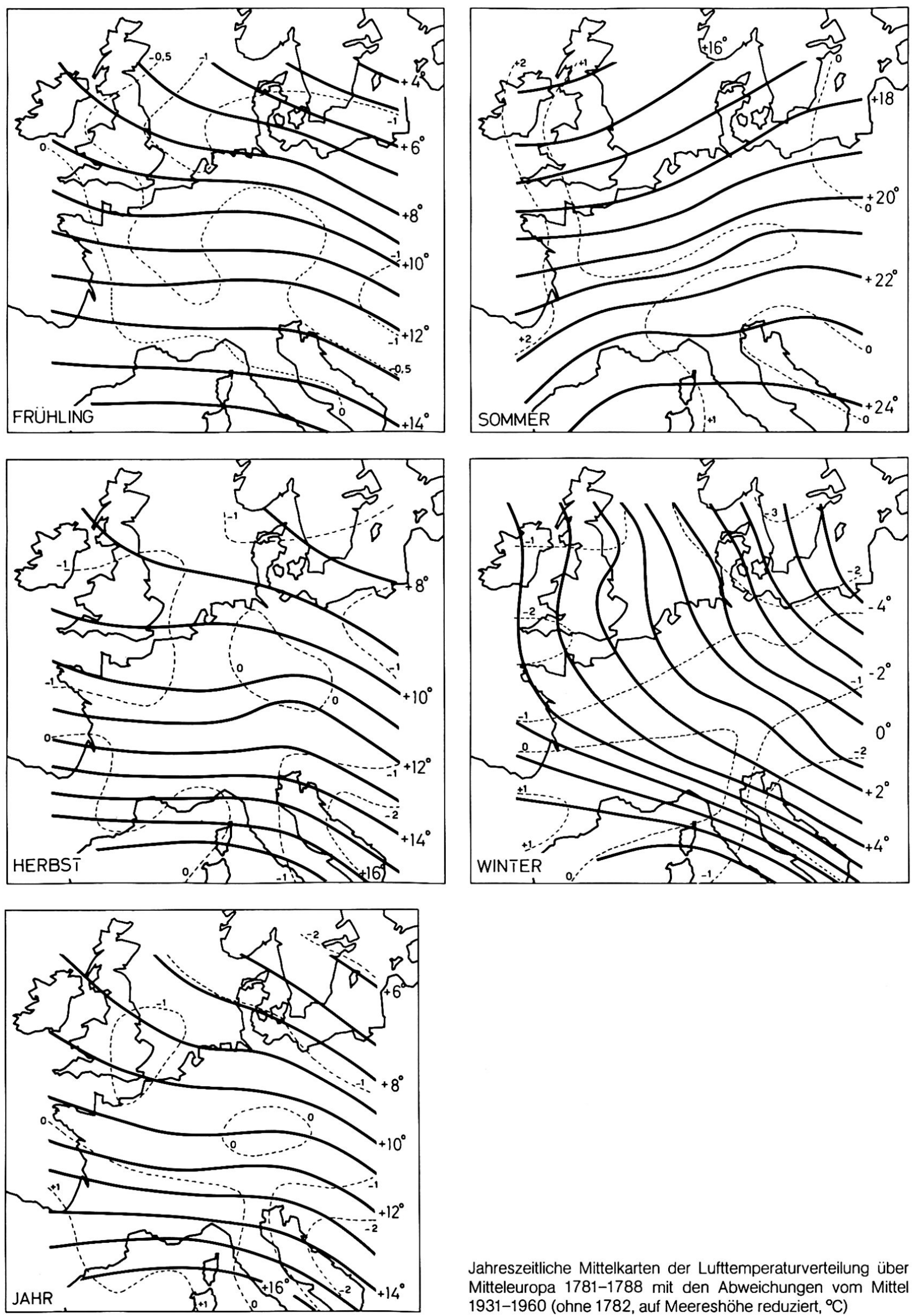

Jahreszeitliche Mittelkarten der Lufttemperaturverteilung über Mitteleuropa 1781-1788 mit den Abweichungen vom Mittel 1931-1960 (ohne 1782, auf Meereshöhe reduziert, ${ }^{\circ} \mathrm{C}$ ) 

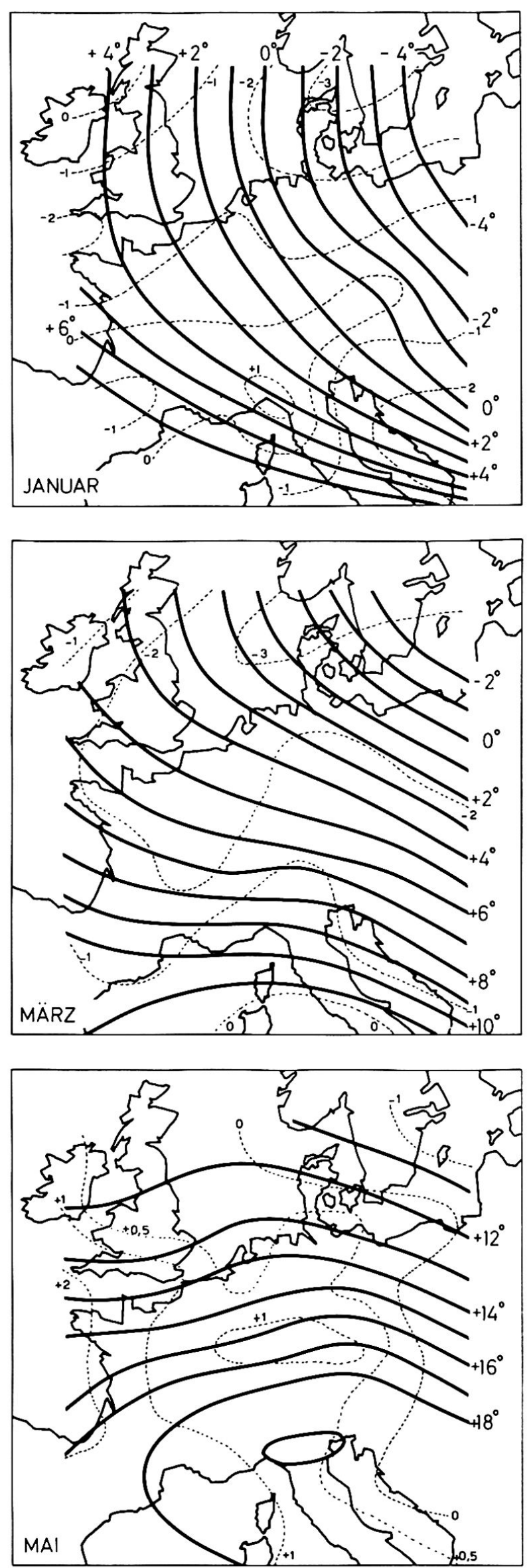
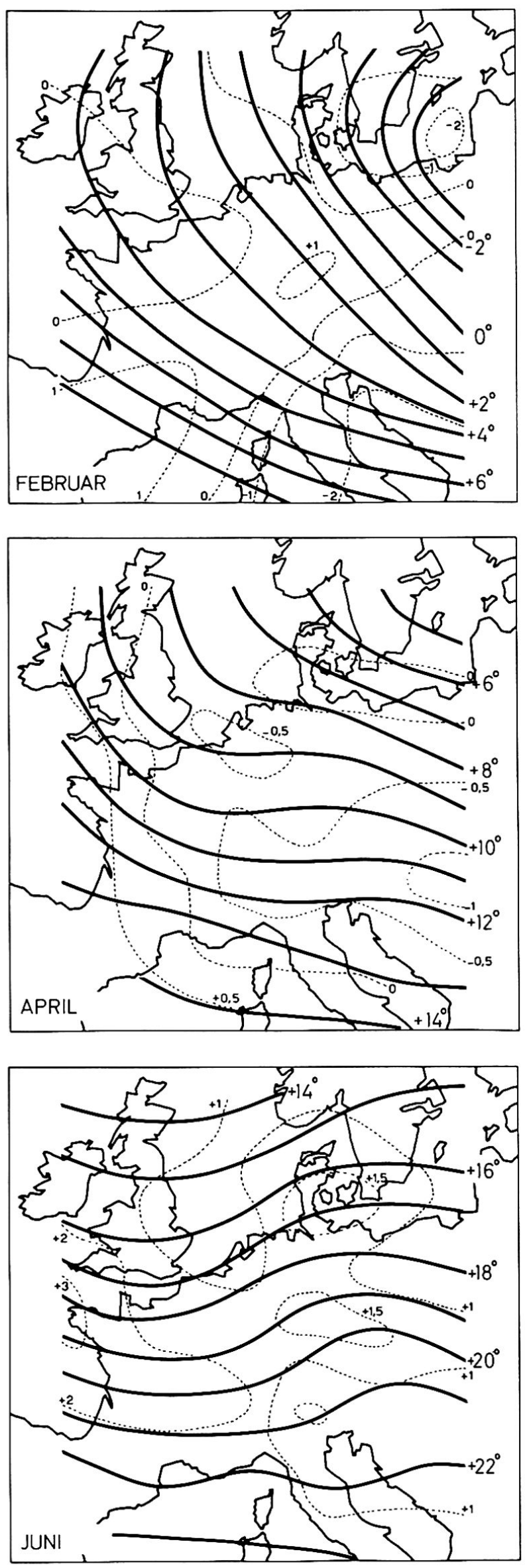

Monatliche Mittelkarten der Lufttemperaturverteilung über Mitteleuropa 1781-1788 mit den Abweichungen vom Mittel 1931-1960 (ohne 1782, auf Meereshöhe reduziert, ${ }^{\circ} \mathrm{C}$ ) 

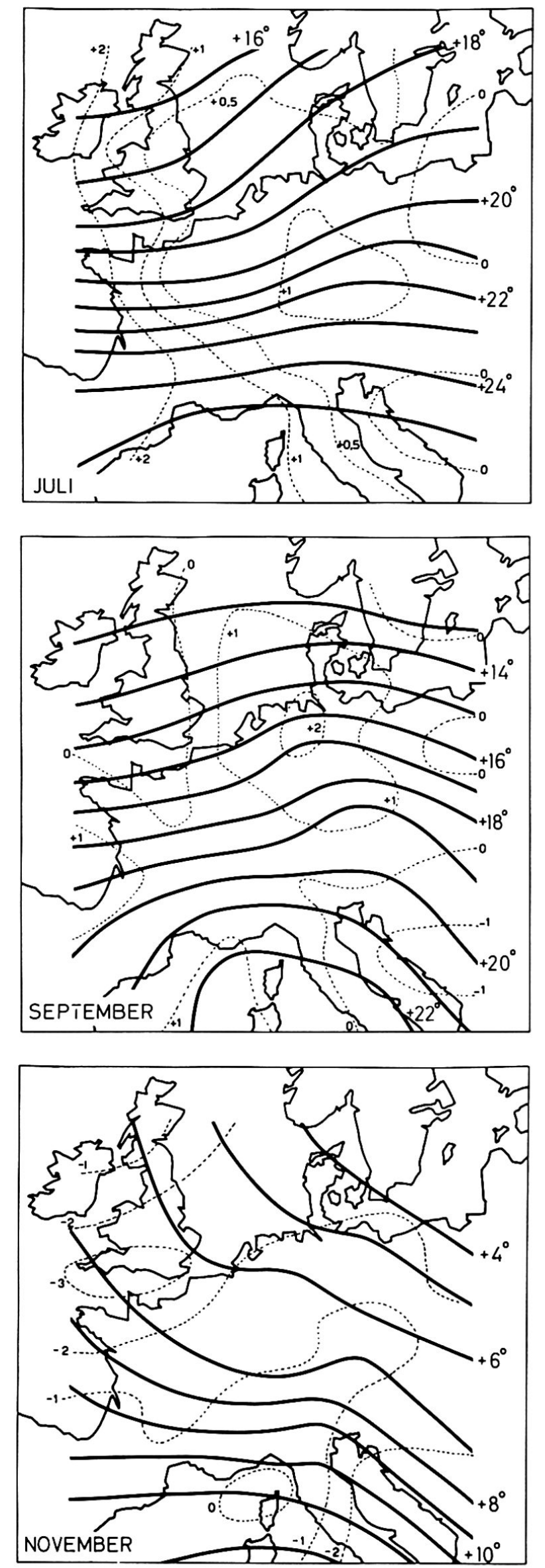
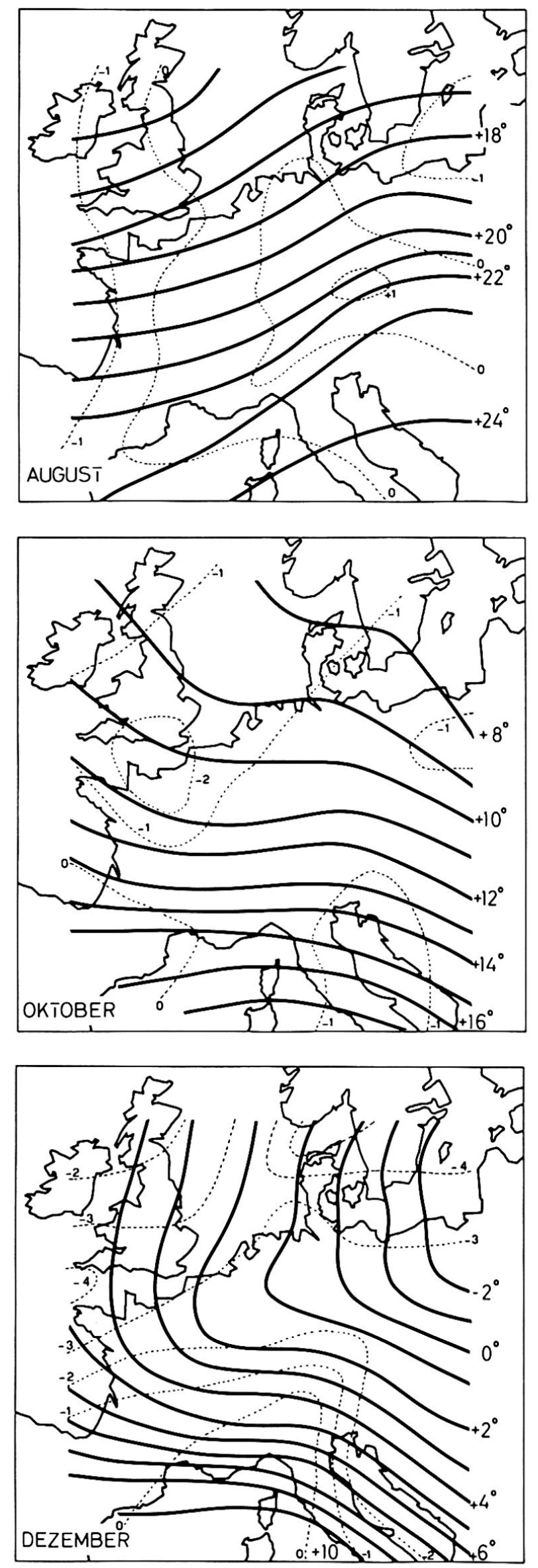


\section{n [Anzahl Fälle]}

10
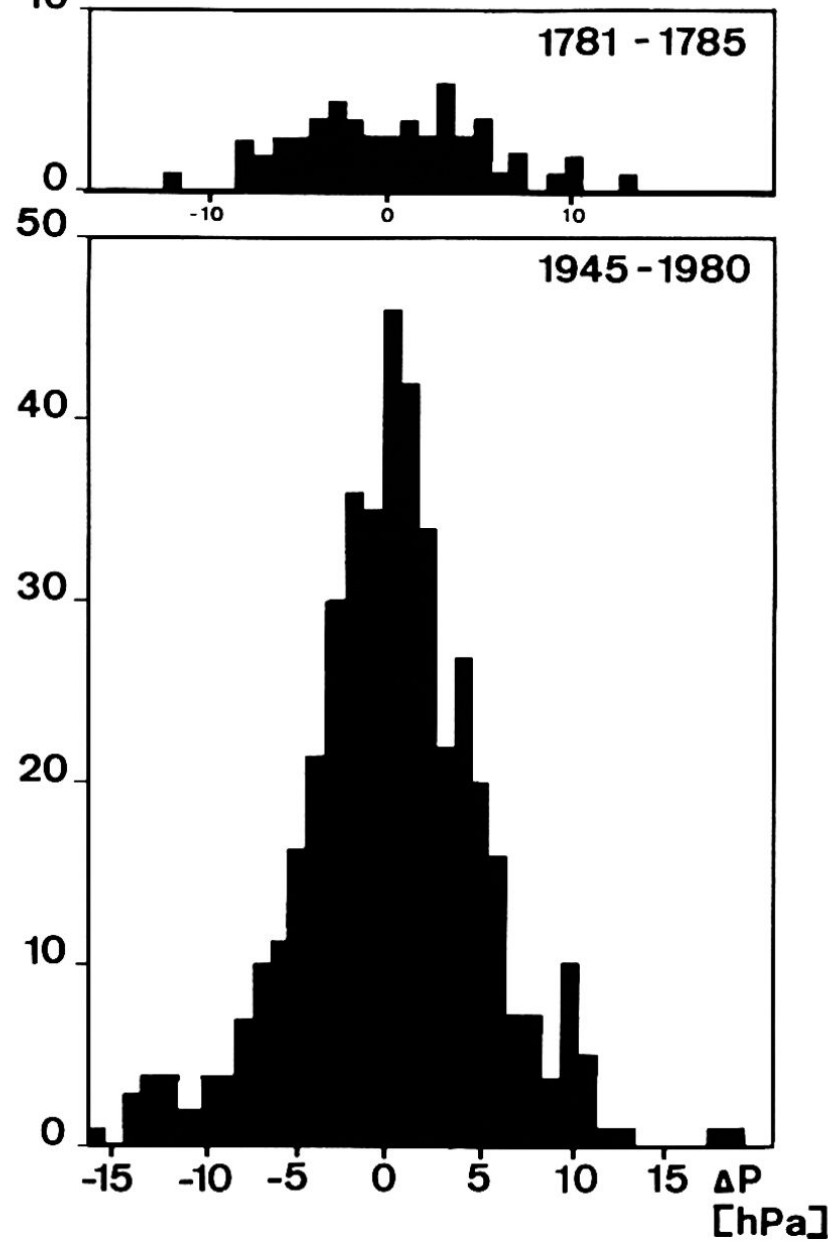

Figur 9 Häufigkeitsverteilungen der monatlichen Luftdruckänderungen über Mitteleuropa 1781-1785 und 1945-1980

Durch die größere südliche Ausdehnung der polaren Vereisung und die Richtung der vorherrschenden atmosphärischen Strömung über dem nördlichen Atlantik dauerte es länger, bis der Kontinent sich temperaturmäßig dem heutigen Sommer-Stand entsprechend erwärmen konnte.

Die seit dem Maunder-Minimum der Sonnenfleckentätigkeit 1640-1715 damals offenbar gekoppelte verminderte Sonnenstrahlungsintensität hat in den 1780 er Jahren noch nicht ihren heutigen Stand erreicht. Dies und der Rückkopplungseffekt einer größeren Albedo kann einerseits bewirkt haben, daß sich das Eis weiter nach Süden ausdehnen konnte. Andererseits wäre die vorindustriell noch klarere Luft vor allem im Sommer für eine Erwärmung auf das heutige Niveau trotz leicht eingeschränkter Sonneneinstrahlung verantwortlich.

Wenn wir das Dezennium 1781-1790 im einzelnen betrachten, fallen zwei markante Monate auf, welche aus dem allgemeinen Rahmen fallen. Sie sind charakteristisch für die damals herrschenden klimatischen Zustände aufgrund der im Atlantik weit nach Süden vorgeschobenen Packeisfläche des Nordpolarmeeres (Figur 11).

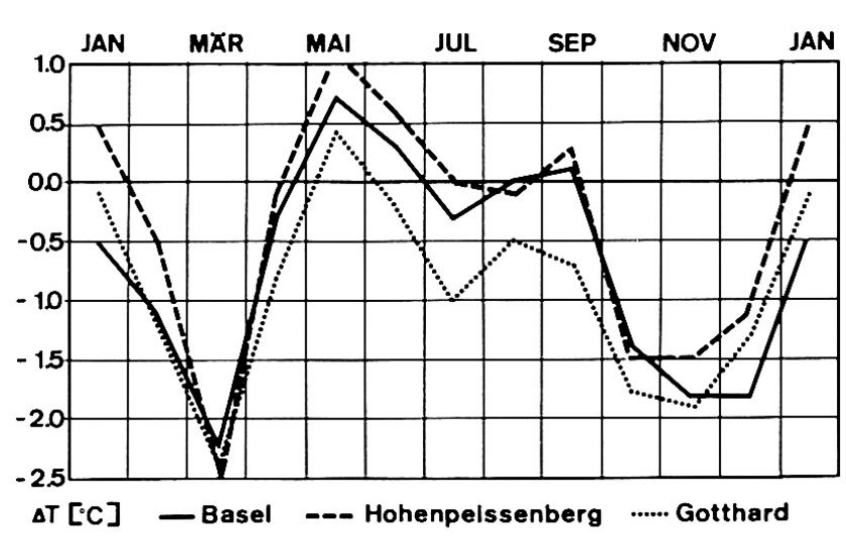

Figur 10 Abweichungen der Lufttemperatur 1781-1790 vom Mittel 1951-1980 im Jahresverlauf

Es handelt sich zunächst um den März 1785, welcher nach den Temperaturmessungen der Basler Reihe die tiefsten Märztemperaturen seit 1755 brachte $\left(-2,8^{\circ} \mathrm{C}\right.$ statt des von 1951-1980 gemessenen Mittelwertes von $5,5^{\circ} \mathrm{C}$; sCHÜEPP, 1961). Da wir für die Jahre 1781-1785 die täglichen KINGTON-Karten besitzen, lassen sich die witterungsklimatologischen Vorgänge in jenem spätwinterlichen Kältemonat im einzelnen verfolgen, welche zum Monatsmittel der Figur 12 führten. Wir finden während des ganzen Monats hohen Druck nordwestlich von Europa, bis zum 7. März mit Kern über der Nordsee, dann zwischen Großbritannien und Island, meist mit Luftdruckwerten zwischen 1030 und $1035 \mathrm{hPa}$ im Zentrum. Über Nordeuropa herrschte sehr häufig eine Nordströmung aus dem Polargebiet, welche immer wieder Kaltluftschübe bis ins Alpengebiet brachte. In Basel wurden am 29. März morgens ca. -14 bis $-15^{\circ} \mathrm{C}$ gemessen! Der dem Polarkreis nahe kalte Nordatlantik begünstigte den blockierenden Hochdruckaufbau in diesem Gebiet, leitete die in normalen Jahren gegen Großbritannien gerichtete atlantische Westströmung gegen Norden ab und verursachte gleichzeitig den Kaltluftstrom nach Mitteleuropa. Diese Erscheinung ist nicht auf den März 1785 beschränkt. Die von KINGTON bearbeitete Periode 1781-1785 ist nach LAMB (1979) charkaterisiert durch auffallend geringe Westwindhäufigkeit im britischen Raum.

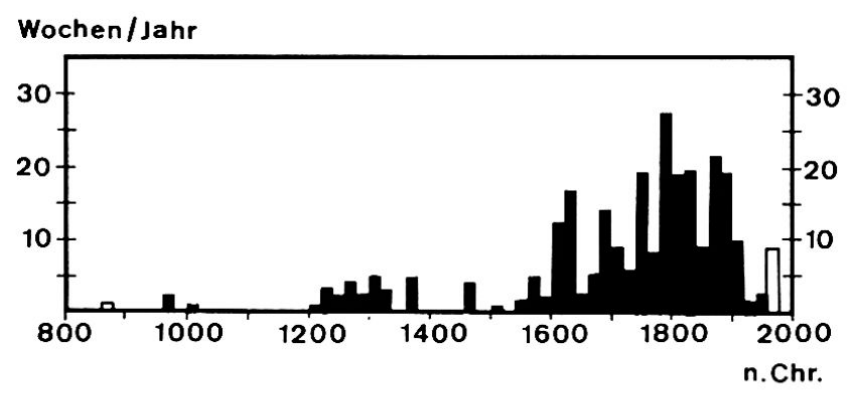

Figur 11 Auftreten von arktischem Meereis an der Küste Islands (nach LAMB, 1982) 


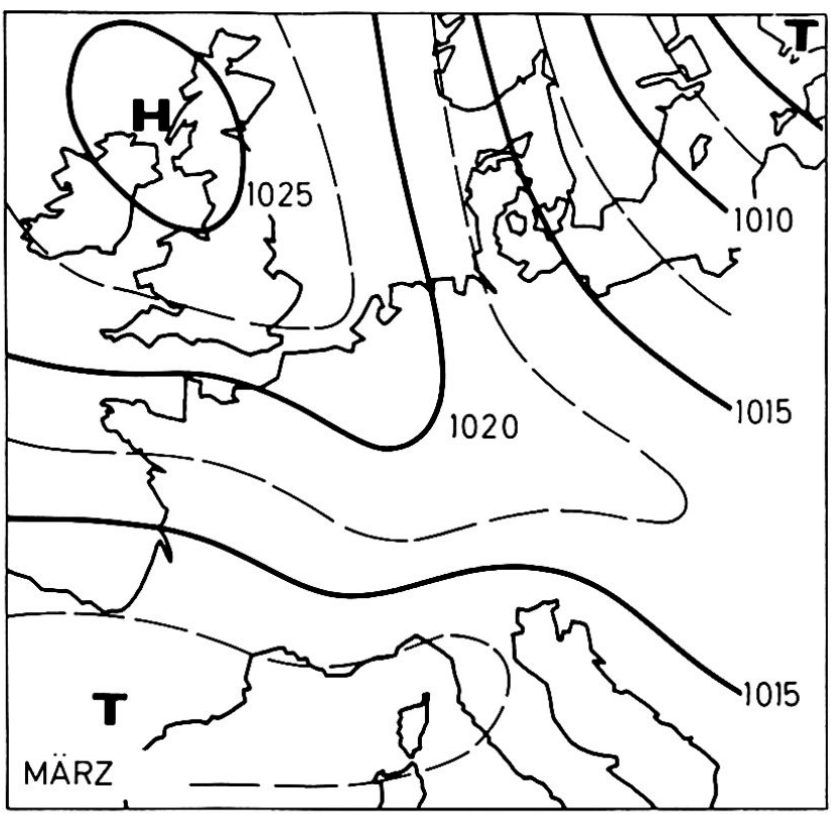

Figur 12 Die mittlere Luftdruckverteilung über Mitteleuropa im März 1785 (auf Meereshöhe reduziert)

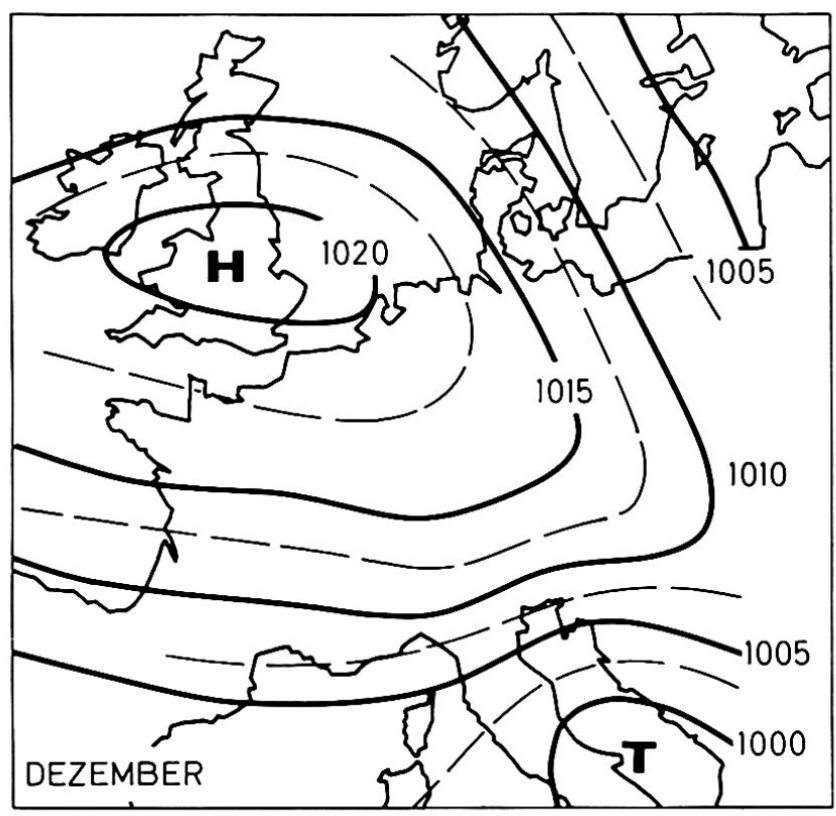

Figur 13 Die mittlere Luftdruckverteilung über Mitteleuropa im Dezember 1788 (auf Meereshöhe reduziert)

Tabelle 4 Temperaturabweichungen vom Mittel 1951-1980 für Basel-Binningen und St. Gotthard

Basel-Binningen

Jahr Jan. Feb. März April Mai Juni Juli Aug. Sept. Okt. Nov. Dez. Jahr
Mittel 1951-1980:

\begin{tabular}{rrrrrrrrrrrrrrr} 
& 0,5 & 2,1 & 5,5 & 9,0 & 13,2 & 16,6 & 18,5 & 17,6 & 14,6 & 9,7 & 4,9 & 1,6 & 9,5 \\
\hline 1781 & $-0,3$ & 0,7 & 0,2 & 2,3 & 1,9 & 0,5 & 0,0 & 2,0 & 0,9 & $-1,8$ & $-0,6$ & 0,9 & 0,6 \\
1782 & 1,7 & $-6,7$ & $-2,0$ & $-1,3$ & $-0,9$ & 1,2 & 1,2 & $-0,1$ & $-0,6$ & $-3,1$ & $-4,4$ & - & 3,0 & $-1,5$ \\
1783 & 3,3 & 0,9 & $-2,0$ & 0,6 & 1,2 & 0,4 & 1,8 & 0,3 & 0,0 & 0,4 & $-0,9$ & - & 2,2 & 0,3 \\
1784 & $-4,4$ & $-4,5$ & $-2,0$ & $-2,6$ & 2,7 & 0,9 & 0,1 & $-1,0$ & 1,6 & $-4,3$ & $-0,9$ & - & 3,2 & $-1,5$ \\
1785 & $-0,4$ & $-4,1$ & $-8,3$ & $-3,0$ & 0,9 & $-0,6$ & $-1,2$ & $-1,0$ & 1,7 & $-1,2$ & $-0,5$ & - & 0,9 & $-1,6$ \\
1786 & $-0,3$ & $-0,5$ & $-3,6$ & 1,5 & $-0,2$ & 1,4 & $-1,9$ & $-1,0$ & $-1,5$ & $-3,3$ & $-2,7$ & - & 0,7 & $-1,1$ \\
1787 & $-3,4$ & $-0,5$ & 0,9 & $-0,7$ & $-1,9$ & 0,7 & $-1,0$ & 0,8 & - & 0,4 & 1,4 & $-0,3$ & 2,3 & $-0,2$ \\
1788 & 0,6 & 1,7 & 0,1 & 0,8 & 1,6 & 0,6 & 1,0 & $-0,4$ & 1,4 & $-1,4$ & $-4,6$ & $-11,2$ & $-0,8$ \\
1789 & $-2,3$ & 1,1 & $-4,4$ & 0,9 & 2,2 & $-2,3-1,3$ & 0,1 & $-1,0$ & $-1,2$ & $-1,9$ & - & 0,1 & $-0,9$ \\
1790 & 0,2 & 0,6 & $-0,7$ & $-1,0$ & 1,1 & $0,4-1,7$ & 0,8 & $-1,3$ & 1,0 & $-0,7$ & 0,5 & $-0,1$ \\
1791 & 2,6 & $-1,4$ & $-1,1$ & 2,7 & $-0,2$ & $-0,1$ & $-0,3$ & 1,6 & $-0,9$ & $-0,6$ & $-3,5$ & 0,5 & $-0,1$ \\
1792 & 0,4 & $-1,0$ & 0,4 & 1,8 & $-0,6$ & $-0,1$ & $-0,5$ & 0,9 & $-1,9$ & 0,6 & $-1,4$ & 0,0 & $-0,1$ \\
\hline
\end{tabular}

Mittlere Abweichung 1781-1790:

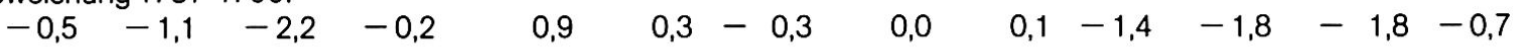

Mittlere Abweichung 1781-1788:

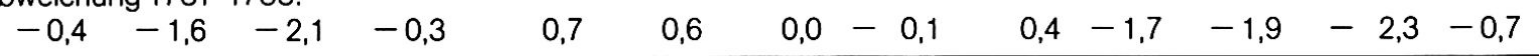

St. Gotthard

Jahr Jan. Feb. März April Mai Juni Juli Aug. Sept. Okt. Nov. Dez. Jahr
Mittel 1951-1980:

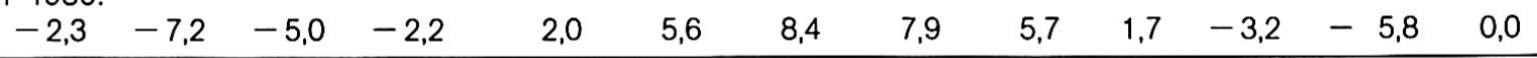

Mittlere Abweichungen 1781-1790:

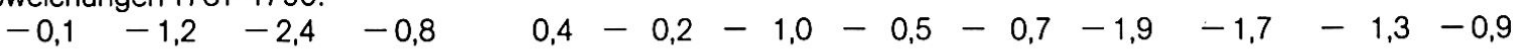

Mittlere Abweichung 1781-1788:

$$
\begin{array}{lllllllllllllll}
0,0 & -1,5 & -2,0 & -0,8 & 0,0 & 0,2 & -0,7 & -0,8 & -0,4 & -2,5 & -1,8 & - & 1,2 & -0,9
\end{array}
$$


Das zweite Hauptereignis der Periode war der ungewöhnlich strenge Frühwinter des Dezembers 1788 mit seinem Basler Temperaturmittel von $-9,6^{\circ} \mathrm{C}$, der zwar vom Dezember 1879 mit $-10,1^{\circ} \mathrm{C}$ noch etwas überboten wurde. In den Alpen brachte dieser Dezember 1788 mit $-12,8^{\circ} \mathrm{C}$ auf dem Gotthard das Dezemberminimum der Temperatur seit Beginn der Messungen in solcher Meereshöhe. Das in Figur 13 wiedergegebene Monatsmittel der Luftdruckverteilung zeigt ähnlich wie der März 1785 ein ausgeprägtes Hoch über den Britischen Inseln. Wir können in beiden Fällen die starke Eisbildung im Nordatlantik mit Absenkung der Wassertemperaturen für die Tendenz zur Ausbildung von starken Hochdruckzentren über Nordwesteuropa als bestimmenden Faktor betrachten. Zusätzlich ist für die allgemeine Senkung des Temperaturniveaus über Mitteleuropa in den 1780er Jahren der Ausbruch des Eldeijars bei Island (1783) als Mithelfer heranzuziehen (Tabelle 4).

Auch wenn der Einfluß der vulkanischen Trübung wohl nur für die besonders kalten Jahre 1784 und 1785 , kaum aber für die erneute Kältewelle des Winters 1788/89 anzunehmen ist, zeigt doch das «Großereignis» des 19. Jahrhunderts, der Ausbruch des weit entfernteren Tambora (Sunda-Archipel, 1815) mit dem nachfolgenden "Jahr ohne Sommer 1816", daß große Staubtrübungen weltweite Auswirkungen haben können. Sie sind, wie die statistischen Berechnungen von SCHÖNWIESE 1983 und 1984 zeigen, nicht allein bestimmendes Signal, sondern haben nur katalytische Wirkung. Die nach Figur 11 bis gegen das Ende des 18. Jahrhunderts zunehmende Vereisung läßt sich kaum durch die Vulkantrübungen erklären. Als letztlich bestimmender Faktor bleibt wohl nur die Annahme einer etwas verringerten Sonnenstrahlung.

In der heutigen Zeit sind die großen Kälteeinbrüche aus dem Polarraum, wie sie im März 1785 und Dezember 1788 auftraten, seltener geworden. Das Packeis ist stark zurückgegangen. Dagegen erobert sich die Kaltluft des nordrussischen Raumes immer noch zeitweise den Weg nach Mitteleuropa, indem sie auf der Südostseite eines skandinavischen Hochs nach Westen vordringt und die atlantischen Luftmassen in den Mittelmeerraum abdrängt, wie es im sehr kalten Februar 1956 der Fall war.

Die Tabelle 4 zeigt uns, daß nach den Kältenachwehen des Jahres 1788 mit einem nochmaligen kalten März und einem kühlen Juni im Jahr 1789 eine Erwärmung einsetzte, daß die Jahre 1790, 1791 und 1792 verhältnismäßig ruhige Witterung im Alpengebiet brachten, während anderseits im Nordseeraum nach LAMB (1984) 1792 heftige Stürme auftraten. Die Dendrochronologie zeigt nach SCHWEINGRUBER (1983) in der Periode ca. 1770-1800 in der Schweiz große Holzzuwachsraten, ein Zeichen für günstige
Spätsommerwitterung bis zum Beginn des 19. Jahrhunderts.

Zum Schluß sei in den Abbildungen 14 und 15 auf den Klimaverlauf seit dem 18. Jahrhundert hingewiesen, der nach kräftigen Temperaturausschlägen im 19. Jahrhundert in der Zeit von etwa 1895-1945 eine markante Sichtverschlechterung zeigt (Figur 14). Dies war verbunden mit einem anhaltenden Temperaturanstieg. Seither ist wieder eine Stagnation festzustellen (Figur 15). Auf die näheren Ursachen dieser Entwicklung soll in einem späteren Artikel eingegangen werden.

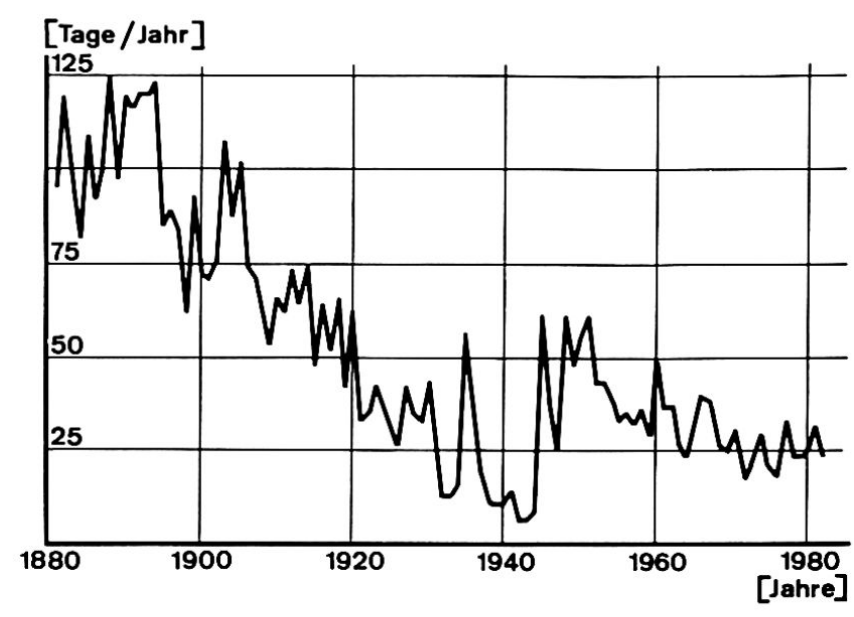

Figur 14 Die Sichtbarkeit der Alpen von Zürich aus ab 1880 (nach GRAF, 1984)

Es ist zu hoffen, daß mittels großräumiger Luftdruckkarten sowie den entsprechenden Niederschlags- und Temperaturabweichungen für den ganzen Zeitraum seit 1781 in den kommenden Jahren die Häufigkeit der einzelnen Witterungslagen ermittelt werden kann. LAMB und JOHNSON haben 1966 den Anstoß dazu gegeben. FLIRI (1984) hat für das Alpengebiet die heutigen Verhältnisse näher untersucht (vgl. Literaturbesprechung Seite 230). Klimahistoriker - hoffentlich nicht bald historische Klimatologen - und andere Wissenschafter in verwandten Gebieten arbeitend, hätten ein 200jähriges Eichmaterial für die Entwicklung von Klimamodellrechnungen mit Computer zur Verfügung. Es ist somit weiterhin wünschenswert - im Hinblick auf eine den Menschen einschließende Ursachendiskussion sogar unbedingt nötig -, eine vertiefte Kenntnis der Ursachen und Wirkungen der «Kleinen Eiszeit» anzustreben und so den noch hängigen Fragen sicherer und näher auf den Leib zu rücken. 


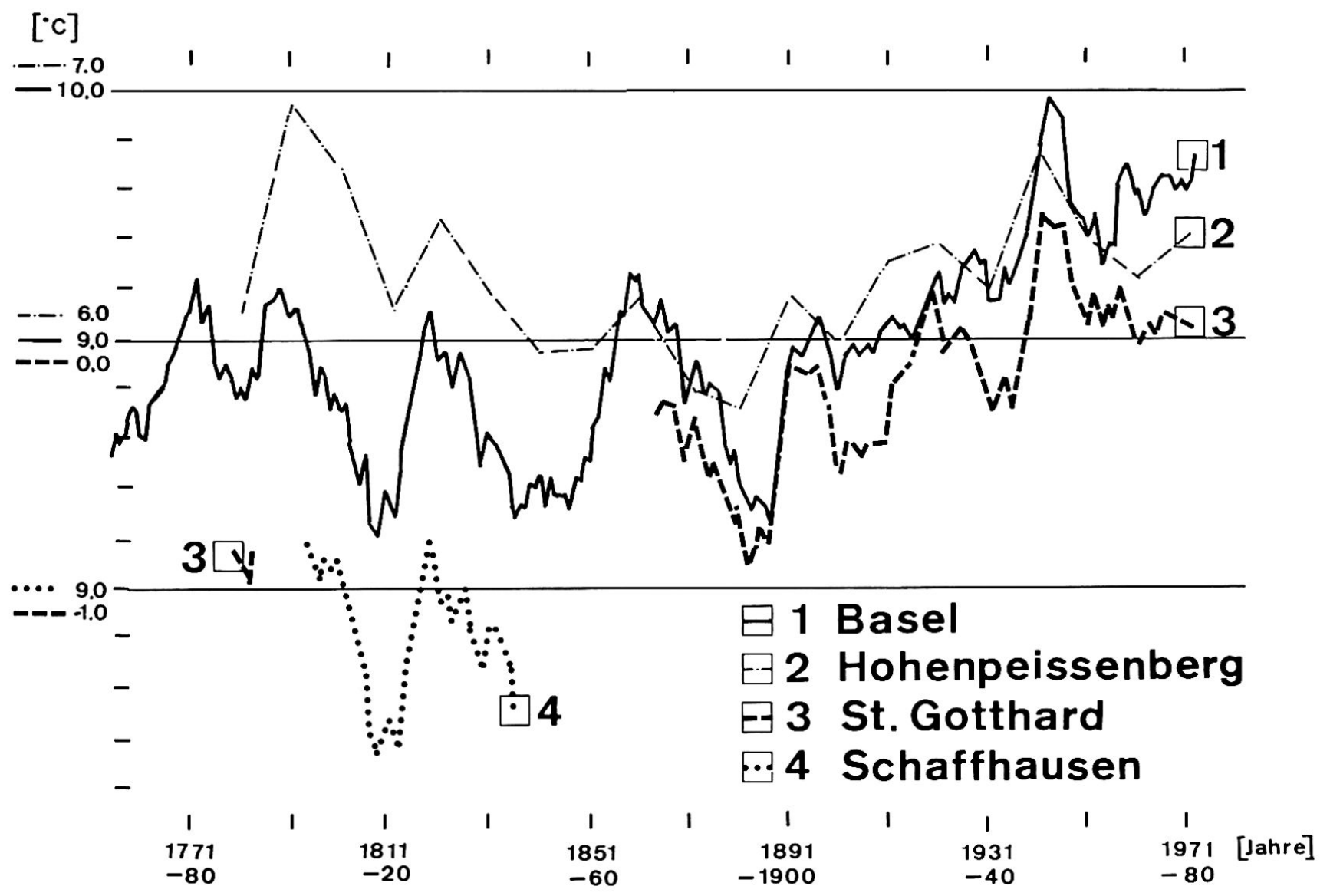

Figur 15 Der Temperaturverlauf 1755-1980 an einigen Meßorten im weiteren Alpenbereich (Gleitende Zehnjahresmittel, Dezennienmittel für den Hohenpeißenberg)

\section{Dank}

Meinen besonderen Dank möchte ich den nachfolgend aufgeführten Einzelpersonen und Institutionen aussprechen für ihre große und bereitwillige Unterstützung mit Idee, Wort und Tat:

Prof.Dr. G.Gensler, Schweiz. Meteorologische Anstalt, Zürich.

Prof. Dr. M. Schüepp, Wallisellen.

Prof. Dr. H. H. Lamb, Climatic Research Unit, University of East Anglia, Norwich.

Schweizerische Meteorologische Anstalt, Zürich.

Zentralbibliothek Luzern.

Die Arbeit wurde ermöglicht durch die finanzielle Unterstützung des Schweizerischen Nationalfonds zur Förderung der wissenschaftlichen Forschung, Bern.

\section{Literatur}

ANNALEN, (1864ff.): Annalen der Schweizerischen Meteorologischen Anstalt, Zürich.

ATTMANNSPACHER, W. (1981): 200 Jahre meteorologische Beobachtungen auf dem Hohenpeißenberg 1781-1980, Berichte des Deutschen Wetterdienstes, Nr. 155, Selbstverlag, Offenbach am Main.

BEHRENS, A. (1965): Die zweihundertjährige Luftdruckreihe von Mailand 1763-1962, Dissertation Universität Zürich.

BIDER, M., SCHÜEPP, M., RUDLOFF, H. von (1959): Die Reduktion der 200jährigen Basler Temperaturreihe. In: Archiv für Meteorologie, Geophysik und Bioklimatologie, Serie B, Vol. 9, Heft 3/4, p. 360-412, Springer, Wien.

BIDER, M., SCHÜEPP, M. (1961): Luftdruckreihen der letzten zwei Jahrhunderte von Basel und Genf, Archiv für Meteorologie, Geophysik und Bioklimatologie, Serie B, Vol.11, Heft 1, Springer, Wien.

BRANDES, H.W. (1820): Untersuchungen über den mittleren Gang der Wärmeänderungen durchs ganze Jahr, J.A. Barth, Leipzig. 
CLAYTON, H. H. (1927/1934): World weather records, Smithsonian Miscellaneous Collections, Vol. 79 und 90, Washington.

EPHEMERIDEN (1781-92): Ephemerides Societatis Meteorologicae Palatinae, Mannheim.

FAY, R. (1958): Some variations in European climatic temperatures. In: Journal of Meteorology, vol. 15, Nr.5, October, p. 467-474.

FLIRI, F., SCHÜEPP, M. (1984): Synoptische Klimatographie der Alpen zwischen Mont Blanc und Hohen Tauern, Wissenschaftliche Alpenvereinshefte, Heft 29, Innsbruck.

GISLER, O. (1983): Die meteorologischen Beobachtungen von Schaffhausen (1794-1845) und Zürich (1767-1802) nebst einigen Betrachtungen über historische Niederschlagsreihen, Physische Geographie, Vol.12, Geographisches Institut der Universität Zürich.

GRAF, A. (1984): Die Alpensicht von Zürich seit 1865 und ihre Abhängigkeit von Wetterlage, Tages- und Jahreszeit, Diplomarbeit, ausgeführt am Geographischen Institut der Universität Zürich.

GREBE, H. (1957): Temperaturverhältnisse des Observatoriums Hohenpeißenberg. In: Das Observatorium Hohenpeißenberg 1781-1955, Berichte des Deutschen Wetterdienstes, Nr.36, Band 5, p. 12-39, Bad Kissingen.

HOLZHAUSER, H. (1984): Zur Geschichte der Aletschgletscher und des Fieschergletschers, Physische Geographie, Vol.13, Geographisches Institut der Universität Zürich.

jONES, P.D., WIGLEY, T.M.L., BRITTA, K.R. (1983): Reconstructing surface pressure patterns using principal components regression on temperature and precipitation data, International Meeting on statistical climatology, 26.-30. Sept. Centro nacional de meteorologia e geofisica, Lisboa.

KEIL, K. (1983): 200jährige meteorologische Beobachtungen auf dem Hohenpeißenberg. In: Meteorologische Rundschau, Jahrgang 36, Heft 5.

KREIL, K. (1854): Mehriährige Beobachtungen in Wien. In: Jahrbücher der Königl. und Kaiserl. Centralanstalt für Meteorologie und Erdmagnetismus, 1. Band, Jahrgang 1848 und 1849, Wien.

KINGTON, J.A. (1978): Historical daily synoptic weather maps for the 1790's. In: Journal of Meteorology, vol. 3, Nr. 27, März, p. 65-71.
LAMB, H. H., JOHNSON, A.I. (1966): Secular variations of the atmospheric circulation since 1750, Geophysical Memoires, vol. XIV, Nr. 110, Meteorological Office, HMSO, London.

LAMB, H.H. (1977): Climate: Present, Past and Future, Vol. 2 Climatic History and the Future, London, Methuen.

LAMB, H. H., WEISS, I. (1979): On recent changes of the wind and wave regime of the North Sea and the outlook, Amt für Wehrgeophysik, Fachliche Mitteilungen, Nr.194, März 1979, Traben-Trarbach.

LAMB, H.H. (1982): Climatic changes in our own times and future threats. In: Geography, vol. 67, Part 3, July, p. $203 \mathrm{ff}$.

LAMB, H.H. (1984): Some studies of the Little Ice Age of recent centuries and its great storms. In: Climatic changes on a yearly to millennial basis, p. 309-329, Reidel, London.

LAUSCHER, F. (1980): Die Wärmeinsel des österreichischen Donauraumes zur Regierungszeit Kaiser Franz Josephs II., Annalen der Meteorologie, Neue Folge, Nr.16, p.199/200, Offenbach.

LAUSCHER, F. (1981): Säkuläre Schwankungen der Dezennienmittel und extreme Jahreswerte der Temperatur in allen Erdteilen, Zentralanstalt für Meteorologie und Geodynamik in Wien, Publ. Nr. 252, Heft 48.

ÖSCHGER, H., MESSERLI, B., SVILAR, M. (1980): Das Klima, Analysen und Modelle, Geschichte und Zukunft, Springer, Berlin.

PFISTER, CHR. (1984): Klimageschichte der Schweiz 1525 bis 1860. Das Klima der Schweiz 1525 bis 1860 und seine Bedeutung in der Geschichte von Bevölkerung und Landwirtschaft, Band 1, Haupt, Bern.

RENOU, M. E. (1881): Etudes sur le climat de Paris. In: Annales du bureau central météorologique de France, Jahrgang 1880 , Paris.

RUDLOFF, H. von (1967): Die Schwankungen und Pendelungen des Klimas in Europa seit dem Beginn der regelmäßigen Instrumentenbeobachtungen (1670), Die Wissenschaft, Band 122, Vieweg, Braunschweig.

SCHÜEPP, M. (1961): Lufttemperatur, 2. Teil, Klimatologie der Schweiz, Heft 2/C, Beiheft zu den Annalen Jahrgang 1960 der Schweizerischen Meteorologischen Anstalt, Zürich, p. 25-27.

SCHWEINGRUBER, F. H. (1983): Der Jahrring, Standort, Methodik, Zeit und Klima in der Dendrochronologie, Haupt, Bern. 